\title{
ON A SEMI-RIGIDITY PROPERTY FOR HOLOMORPHIC MAPS*
}

\author{
XIAOJUN HUANG ${ }^{\dagger}$
}

Dedicated to Professor Yum-Tong Siu on the occasion of his 60th birthday

1. Introduction. In this paper, we are concerned with a rigidity problem for holomorphic maps between balls in complex spaces of different dimensions. Write $\mathbf{B}^{n}$ and $\mathbf{B}^{N}$ for the unit balls in $\mathbf{C}^{n}$ and $\mathbf{C}^{N}$, respectively. Let $F$ be a proper holomorphic map from $\mathbf{B}^{n}$ into $\mathbf{B}^{N}$. We say that $F$ is $\kappa$-linear if for any point $p \in \mathbf{B}^{n}$, there is an affine complex subspace $S_{p}^{\kappa}$, that passes through $p$ and is of dimension $\kappa$, such that for any affine complex line $L$ contained in $S_{p}^{\kappa}, F\left(L \cap \mathbf{B}^{n}\right)$ is contained in an affine complex line in $\mathbf{C}^{N}$. Our main result is the following:

THEOREM 1.1. Let $F$ be a proper holomorphic map from $\mathbf{B}^{n}$ into $\mathbf{B}^{N}$, which is $C^{3}$ smooth up to the boundary. Write $P(n, \kappa)=\frac{\kappa(2 n-\kappa-1)}{2}$. If $1 \leq \kappa \leq n-1$ and $N-n<P(n, \kappa)$, then $F$ is $(n-\kappa+1)$-linear.

For an affine complex subspace $S \subset \mathbf{C}^{n}$ of dimension at least 2 and a proper holomorphic map $F$ from $\mathbf{B}^{n}$ into $\mathbf{B}^{N}, F$ maps each affine complex disk in $S \cap \mathbf{B}^{n}$ to an affine complex disk in $\mathbf{B}^{N}$ if and only if the restriction of $F$ to $S$ is a linear fractional embedding to its image $([\mathrm{Alx}])$. As a consequence, Theorem 1.1 is equivalent to the following:

TheOREM 1.2. Let $F$ be a proper holomorphic map from $\mathbf{B}^{n}$ into $\mathbf{B}^{N}$, which is $C^{3}$-smooth up to the boundary. Write $P(n, \kappa)=\frac{\kappa(2 n-\kappa-1)}{2}$. If $1 \leq \kappa \leq n-1$ and $N-n<P(n, \kappa)$, then for any point $p \in \mathbf{B}^{n}$, there are certain $\sigma_{p} \in A u t\left(\mathbf{B}^{n}\right)$ and $\tau_{p} \in$ $A u t\left(\mathbf{B}^{N}\right)$ with $\sigma_{p}(0)=p$ and $\tau_{p}(F(p))=0$ such that $\tau_{p} \circ F \circ \sigma_{p}\left(z_{1}, \cdots, z_{n-\kappa+1}, 0, \cdots, 0\right)$ $=\left(z_{1}, \cdots, z_{n-\kappa+1}, 0, \cdots, 0\right)$.

The following example shows that in Theorem 1.1, when $N-n \geq P(n, \kappa)$, one can not expect the $(n-k+1)$-linearity for the map in general.

EXAMPLE 1.3. Let

$$
\begin{aligned}
& \psi_{1}=\left(z_{1}^{2}, \sqrt{2} z_{1} z_{2}, \cdots, \sqrt{2} z_{1} z_{k-1}, z_{1} z_{k}, \cdots, z_{1} z_{n}\right), \\
& \psi_{2}=\left(z_{2}^{2}, \sqrt{2} z_{2} z_{3}, \cdots, \sqrt{2} z_{2} z_{k-1}, z_{2} z_{k}, \cdots, z_{2} z_{n}\right), \\
& \cdots \\
& \psi_{k-1}=\left(z_{k-1}^{2}, z_{k-1} z_{k}, \cdots, z_{k-1} z_{n}\right) \\
& \psi_{k}=\left(z_{k}, \cdots, z_{n}\right) .
\end{aligned}
$$

Let $W_{n, k}=\left(\psi_{1}, \cdots, \psi_{k}\right)$. Then $W_{n, k}$ is a proper polynomial map from $\mathbf{B}^{n}$ into $\mathbf{B}^{N}$ with $N=n+P(n, k)$. Notice that $W_{n, k}$ is not $(n-k+1)$-linear.

*Received November 23, 2002; accepted for publication July 7, 2003.

†Department of Mathematics, Rutgers University, New Brunswick, NJ 08903, USA (huangx@math.rutgers.edu). Supported in part by NSF-0200689 and a grant from the Rutgers University Research Council. 
REMARK 1.4. When $N<\frac{n(n+1)}{2}$ in Theorem 1.1, our result says that at each point in the ball, $F$ has at least two independent directions along which the map is linear. When $N \geq \frac{n(n+1)}{2}, F$ usually has no partial linearity. To see this, we just need to notice that the polynomial map that sends

$$
\begin{aligned}
& \left(z_{1}, \cdots, z_{n}\right) \text { to } \\
& \left(z_{1}^{2}, \sqrt{2} z_{1} z_{2}, \cdots, \sqrt{2} z_{1} z_{n}, z_{2}^{2}, \sqrt{2} z_{2} z_{3}, \cdots, \sqrt{2} z_{2} z_{n}, \cdots, z_{n-1} z_{n-1}, \sqrt{2} z_{n-1} z_{n}, z_{n}^{2}\right)
\end{aligned}
$$

is proper from $\mathbf{B}^{n}$ into $\mathbf{B}^{N}$ with $N=n+P(n, n-1)=\frac{n(n+1)}{2}$. We mention the interesting similarity between the minimal target dimension $N$ for which the rigidity breaks down in the case we are considering here and the minimal target dimension in the classical Cartan-Janet theorem for which there is no more obstruction to locally isometrically embed an analytic Riemannian manifold of dimension $n$ into $\mathbf{R}^{N}$ ([Sp]).

The study of holomorphic maps between balls in complex Euclidean spaces was initiated from a paper of Poincaré [Po], and has attracted considerable attention since then. When $N=n>1$, a result of Alexander [Alx] states that any proper holomorphic self-map of the unit ball $\mathbf{B}^{n}$ in $\mathbf{C}^{n}$ with $n>1$ is an automorphism. Notice that for an affine complex line $L, L \cap \mathbf{B}^{n}$ is a complex geodesic in terms of the hyperbolic Kobayashi metric of the ball and an automorphism of $\mathbf{B}^{n}$ maps an affine line to an affine line. The result of Alexander hence tells that a proper holomorphic self-map of $\mathbf{B}^{n}$ preserves the complex geodesics of $\mathbf{B}^{n}(n>1)$. More generally, one says that a map from $\mathbf{B}^{n}$ into $\mathbf{B}^{N}$ is a linear map or a totally geodesic embedding if it maps a complex geodesic in $\mathbf{B}^{n}$ to a complex geodesic in $\mathbf{B}^{N}$. Webster [We1] [We2] was the first one to look at the geometric structure of proper holomorphic maps between balls in complex spaces of different dimensions. He showed that a proper holomorphic map from $\mathbf{B}^{n}$ into $\mathbf{B}^{n+1}$ with $n>2$, which is three times differentiable up to the boundary, is a totally geodesic embedding. Subsequently, Cima-Suffridge [CS1] reduced the boundary regularity in Webster's theorem to the $C^{2}$-regularity. Motivated by a conjecture posed in [CS1], Faran in [Fa1] showed that any proper holomorphic map from $\mathbf{B}^{n}$ into $\mathbf{B}^{N}$ with $N<2 n-1$, that is analytic up to the boundary, is also a totally geodesic embedding. Forstneric in [Fo1] [Fo2] proved that any proper holomorphic map from $\mathbf{B}^{n}$ into $\mathbf{B}^{N}$ is rational, if the map is $C^{N-n+1}$-regular up to the boundary, which, in particular, reduces the regularity assumption in Faran's linearity theorem to the $C^{N-n+1}$-smoothness.

The structure of the maps gets more complicated when $N \geq 2 n-1$. (See the book [Da1], [BER], and the survey article [Hu2] for more explanations). Recall that two proper holomorphic maps $f, g$ from $\mathbf{B}^{n}$ into $\mathbf{B}^{N}$ are called equivalent if there are $\sigma \in \operatorname{Aut}\left(\mathbf{B}^{n}\right)$ and $\tau \in \operatorname{Aut}\left(\mathbf{B}^{N}\right)$ such that $g=\tau \circ f \circ \sigma$. It is easy to verify that a map is linear if and only if it is equivalent to the standard big circle embedding $L(z): z \rightarrow(z, 0)$. In [Fa2], it was shown that there are four different inequivalent maps from $\mathbf{B}^{2}$ into $\mathbf{B}^{3}$, which are $C^{3}$-smooth up to the boundary. By the work of D'Angelo [Da2], any two proper holomorphic maps from $\mathbf{B}^{n}$ into $\mathbf{B}^{N}$ are homotopically equivalent through a family of inequivalent maps in a suitably larger space, which, in particular, can be used to show that there is a continuous family of inequivalent proper holomorphic quadratic polynomial embeddings from $\mathbf{B}^{n}$ into $\mathbf{B}^{2 n}$. See also [DL] for discussions on the classification of proper monomial maps between balls with certain symmetry. At this point, we mention that the discovery of inner functions can be used to show that there is a proper holomorphic map from $\mathbf{B}^{n}$ into $\mathbf{B}^{n+1}$, which can not be $C^{2}$-smooth at any boundary point. (See [HS], [Low], [For2], [Dor], etc). 
In [Hu1] and, subsequently, a joint paper with Ji [HJ], we considered two natural questions arising from the above mentioned work. In [Hul], we proved that any proper holomorphic map which is only $C^{2}$-regular up to the boundary must be linear if $N<2 n-1$, by applying a different method from the above mentioned work. It is not clear to us if this $C^{2}$-regularity is optimal or not for the super-rigidity to hold, the result in [Hu1] gives a first result in which the required regularity is independent of the codimension. Moreover, some of the basic approaches developed in [Hu1] seem to be quite useful for the study of many other related problems (see [HJ], [EHZ1] [EHZ2]), and will also be used in the present paper. In a joint paper with Ji [HJ], it was shown that any proper holomorphic map from $\mathbf{B}^{n}$ into $\mathbf{B}^{N}$ with $N=2 n-1, n \geq 3$, which is $C^{2}$-smooth up to the boundary, is either linear or equivalent to the Whitney map $W: z=\left(z_{1}, \cdots, z_{n}\right) \rightarrow\left(z_{1}, \cdots, z_{n-1}, z_{n} z\right)$ ([Theorem 1, Theorem 2.3; HJ]). Since the Whitney map is not an immersion, together with the aforementioned work of Faran [Fo2], this shows that any proper holomorphic embedding from $\mathbf{B}^{n}$ into $\mathbf{B}^{N}$ with $N=2 n-1$, which is twice continuously differentiable up to the boundary, must be a linear map.

The present paper continues the work in [Hu1] and [HJ]. Our main result, Theorem 1.1, provides a description of the partial linearity for proper holomorphic maps between balls for $N<n+P(n, n-1)=\frac{n(n+1)}{2}$. When $N \geq n+P(n, n-1)$, the partial linearity breaks down by Remark 1.4. However, there are still many problems left to be understood. We refer the reader to the book of D'Angelo [Da3] for discussions on this matter and we wish at least to mention here a result due to Catlin and D'Angelo [CD] and D'Angelo [Da3], which states that for any polynomial functions $q(z), p_{1}(z)$ with $\left|p_{1}(z)\right|<|q(z)|$ on the closure of $\mathbf{B}^{n}$, there exists a vector valued polynomial $p(z)$ with $N\left(q, p_{1}\right)$ - components such that $\frac{\left(p_{1}(z), p(z)\right)}{q(z)}$ properly holomorphically maps $\mathbf{B}^{n}$ into $\mathbf{B}^{N\left(q, p_{1}\right)}$, where $N\left(q, p_{1}\right)$ depends on $\left(q, p_{1}\right)$ and $N\left(q, p_{1}\right) \rightarrow \infty$ as $\max _{z \in \overline{\mathbf{B}}^{n}}\left|p_{1} / q(z)\right| \rightarrow 1$.

Finally, we mention that in the past years, there has been much work done on various related rigidity problems for holomorphic maps between complex hyperbolic space forms, bounded symmetric domains, etc. To name a few, we refer the reader to the work [We2], [CaMo], [Mok], [MSY], [Tu], [EHZ1], [EHZ2] and the references therein.

Acknowledgment. This paper was written when the author was taking a year long sabbatical leave from Rutgers University at UCSD (Spring, 2002), University of Rouen (Summer, 2002) and The University of Chicago (Fall, 2002). The author would like to thank these Institutes for providing him with an excellent research environment during his stay. He also likes to thank S. Baouendi, P. Ebenfelt, J. D'Angelo, S. Ji, N. Mir, L. Rothschild, S. Webster and D. Zaitsev for their interest to this work. He thanks one of the referees for the very careful reading and many helful suggestions to the paper, which has greatly improved the readability of the paper.

2. Preliminaries, a geometric invariant and partial linearity. Our proof of Theorem 1.1 is based on the approach developed in [Hul] and [HJ]. In this section, we start by recalling some notation, definition and various formulas established in [Hu1] and [HJ], which will be used throughout the paper. Then we introduce a geometric invariant for the map and discuss how it is related to the partial linearity.

2.1. In this subsection, we recall some notation and formulas established in [Hu1] $[\mathrm{HJ}]$. Then we introduce the concept of the geometric rank, which is the invariant 
closely related to the dimension of the affine complex subspaces along which the map is linear.

First, since the ball $\mathbf{B}^{n} \subset \mathbf{C}^{n}$ is equivalent to the Siegel upper-half space, denoted by $\mathbf{S}^{n}:=\left\{(z, w) \in \mathbf{C}^{n-1} \times \mathbf{C}: \operatorname{Im}(w)>|z|^{2}\right\}$, and the punctured sphere is equivalent to the Heisenberg hypersurface, we will mainly focus on mappings between Heisenberg hypersurfaces. In the last section of the paper, we will see how this will immediately give results for mappings between balls by applying the Cayley-type transformations.

Let $M_{1} \subset \mathbf{H}_{n}$ and $M_{2} \subset \mathbf{H}_{N}$ be two connected open pieces of the standard Heisenberg hypersurfaces in $\mathbf{C}^{n}$ and $\mathbf{C}^{N}$, respectively. Here

$\mathbf{H}_{n}:=\left\{(z, w) \in \mathbf{C}^{n}, \operatorname{Im} w=\sum_{j=1}^{n-1}\left|z_{j}\right|^{2}\right\} ; \quad \mathbf{H}_{N}:=\left\{\left(z^{*}, w^{*}\right) \in \mathbf{C}^{N}, \operatorname{Im} w^{*}=\sum_{j=1}^{N-1}\left|z_{j}^{*}\right|^{2}\right\}$.

Write $L_{j}=2 i \overline{z_{j}} \frac{\partial}{\partial w}+\frac{\partial}{\partial z_{j}}$ for $j=1, \cdots, n-1$ and $T=\frac{\partial}{\partial u}$ with $w=u+i v$. Then $\left\{L_{1}, \cdots, L_{n-1}\right\}$ forms a global basis for the complex tangent bundle $\mathrm{T}^{(1,0)} \mathbf{H}_{n}$ of $\mathbf{H}_{n}$, and $T$ is a tangent vector field of $\mathbf{H}_{n}$ transversal to $T^{(1,0)} M \cup T^{(0,1)} M$. Parameterize $\mathbf{H}_{n}$ by $(z, \bar{z}, u)$ through the map $(z, \bar{z}, u) \rightarrow\left(z, u+i|z|^{2}\right)$. In what follows, we will assign the weight of $z$ and $u$ to be 1 and 2, respectively. For a non negative integer $m$, a function $h(z, \bar{z}, u)$ defined over a small ball $U$ of 0 in $\mathbf{H}_{n}$ is said to be of quantity $o_{w t}(m)$, if $\frac{h\left(t z, t \bar{z}, t^{2} u\right)}{|t|^{m}} \rightarrow 0$ uniformly for $(z, u)$ on any compact subset of $U$ as $t(\in \mathbf{R}) \rightarrow 0$. (In this case, we write $h=o_{w t}(m)$. By convention, we write $h=o_{w t}(0)$ if $h \rightarrow 0$ as $(z, \bar{z}, u) \rightarrow 0)$. Also, we write $\chi(z, \bar{z}, u) \in \mathcal{P}+o_{w t}(m)$ if $\chi=h_{1}+h_{2}$ with $h_{1}$ a polynomial in $(z, \bar{z}, u)$ and $h_{2}=o_{w t}(m)$. For a function $h(z, \bar{z}, u)$ defined over $U$, we use $h^{(k)}(z, \bar{z}, u)$ for the sum of terms of weighted degree $k$ in the weighted expansion of $h$ up to order $k$. If $h$ is not specified, we use it to denote a weighted homogeneous polynomial of weighted degree $k$. For a weighted homogeneous holomorphic polynomial of degree $k$, we use the notation: $(\cdot)^{(k)}(z, w)$, or $(\cdot)^{(k)}(z)$ if it depends only on $z$.

For two $m$-tuples $x=\left(x_{1}, \cdots, x_{m}\right), y=\left(y_{1}, \cdots, y_{m}\right)$, we write $(x, y)=x \cdot y^{t}=$ $\sum_{j=1}^{m} x_{j} y_{j}$, and $|x|^{2}=\sum_{j=1}^{m}\left|x_{j}\right|^{2}$.

In all that follows, we assume that $N \geq n>1$. Also, $S \subset \mathbf{C}^{n}$ is called an affine complex subspace of dimension $k$, if $S-p_{0}$ with $p_{0} \in S$ is a $k$-dimensional complex linear subspace of $\mathbf{C}^{n}$.

Let

$$
F=(f, \phi, g)=\left(f_{1}, \cdots, f_{n-1}, \phi_{1}, \cdots, \phi_{N-n}, g\right)
$$

be a non-constant $C^{2}$-smooth CR map from $M_{1}$ into $M_{2}$. Then for each $p \in M_{1}$, we have an associated CR map $F_{p}$ from a small neighborhood of $0 \in \mathbf{H}_{n}$ to $\mathbf{H}_{N}$ with $F_{p}(0)=0$, defined by

$$
F_{p}=\tau_{p}^{F} \circ F \circ \sigma_{p}^{0}=\left(f_{p}, \phi_{p}, g_{p}\right),
$$

where for each $p=\left(z_{0}, w_{0}\right) \in M_{1}$, we write $\sigma_{\left(z_{0}, w_{0}\right)}^{0} \in \operatorname{Aut}\left(\mathbf{H}_{n}\right)$ for the map sending $(z, w)$ to $\left(z+z_{0}, w+w_{0}+2 i<z, \overline{z_{0}}>\right)$ and we define $\tau_{\left(z_{0}, w_{0}\right)}^{F} \in \operatorname{Aut}\left(\mathbf{H}_{N}\right)$ by $\tau_{\left(z_{0}, w_{0}\right)}^{F}\left(z^{*}, w^{*}\right)=\left(z^{*}-\widetilde{f}\left(z_{0}, w_{0}\right), w^{*}-\overline{g\left(z_{0}, w_{0}\right)}-2 i<z^{*}, \widetilde{\widetilde{f}\left(z_{0}, w_{0}\right)}>\right)$, where $\widetilde{f}=(f, \phi)$. 
Let

$$
\lambda(p)=g_{w}^{\prime}(p)-2 i<\tilde{f}_{w}^{\prime}(p), \overline{\widetilde{f}(p)}>=\left|L_{j}(\tilde{f})\right|^{2}
$$

Then under the assumption that $F$ is not a constant map, one has $\lambda(p)>0$. (See [Hu1]).

$$
\text { Let }
$$

$$
\begin{aligned}
& E_{l}(p)=\left.\left(\frac{\partial \tilde{f}_{p}}{\partial z_{l}}\right)\right|_{0}=\left.\left(\frac{\partial f_{p, 1}}{\partial z_{l}}, \cdots \frac{\partial f_{p, n-1}}{\partial z_{l}}, \frac{\partial \phi_{p, 1}}{\partial z_{l}}, \cdots \frac{\partial \phi_{p, N-n}}{\partial z_{l}}\right)\right|_{0}=L_{l}(\widetilde{f})(p) \\
& E_{w}(p)=\left.\left(\frac{\partial \tilde{f}_{p}}{\partial w}\right)\right|_{0}=\left.\left(\frac{\partial f_{p, 1}}{\partial w}, \cdots \frac{\partial f_{p, n-1}}{\partial w}, \frac{\partial \phi_{p, 1}}{\partial w}, \cdots \frac{\partial \phi_{p, N-n}}{\partial w}\right)\right|_{0}=\widetilde{f_{w}^{\prime}}(p)=T(f)(p) .
\end{aligned}
$$

Then the rank of $\left\{E_{1}(p), \cdots, E_{n-1}(p)\right\}$ is $(n-1)$. (See [Hu1]). Let $C_{l}(p)$ be so chosen that (see [pp 17, Hu1])

$$
A(p)=A_{(z, w)}:=\left(\begin{array}{c}
E_{1}(p) / \sqrt{\lambda(p)} \\
\cdot \\
\cdot \\
\cdot \\
E_{n-1}(p) / \sqrt{\lambda(p)} \\
C_{1}(p) \\
\cdots \\
C_{N-n}(p)
\end{array}\right) \text { is a unitary matrix. }
$$

Define $F_{p}^{*}=\left({\widetilde{f_{p}}}^{*}, g_{p}^{*}\right)=\left(\left(f_{p}\right)_{1}^{*}, \cdots,\left(f_{p}\right)_{n-1}^{*},\left(\phi_{p}\right)_{1}^{*}, \cdots,\left(\phi_{p}\right)_{N-n}^{*}, g_{p}^{*}\right)$ by

$$
\frac{1}{\sqrt{\lambda(p)}} F_{p} \cdot\left(\begin{array}{cc}
\overline{A^{t}(p)} & 0 \\
0 & 1 / \sqrt{\lambda(p)}
\end{array}\right) .
$$

Then $F_{p}^{*}$ is still a $C^{2} \mathrm{CR}$ map from a neighborhood of the origin of $\mathbf{H}_{n}$ into $\mathbf{H}_{N}$ with $F_{p}^{*}(0)=0$ and

$$
f_{p}^{*}=z+O\left(|w|+|(z, w)|^{2}\right), \phi_{p}^{*}=O\left(|w|+|(z, w)|^{2}\right), g_{p}^{*}=w+O\left(|(z, w)|^{2}\right) .
$$

As in [Hu1], [HJ], we further modify $F_{p}^{*}$ as follows:

Let

$$
\begin{aligned}
& \mathbf{a}(p):=\frac{\partial{\widetilde{f_{p}}}^{*}}{\partial w}(0)=\left(a_{1}(p), \cdots, a_{n-1}(p), b_{1}(p), \cdots, b_{N-n}(p)\right) \text { with } \\
& a_{l}(p)=\frac{1}{\lambda(p)} E_{w}(p) \cdot \overline{E_{l}(p)} \text { for } l \leq n-1
\end{aligned}
$$

It can be easily seen that

$$
|\mathbf{a}(p)|^{2}=\frac{1}{\lambda(p)}\left|E_{w}(p)\right|^{2}
$$

(see $[\S 2, \mathrm{Hu} 1]))$. Also let 
(2.1.6)

$$
\begin{aligned}
& d_{l j}(p):=\left.\frac{\partial^{2}\left(f_{p}\right)_{l}^{*}}{\partial z_{j} \partial w}\right|_{0}=\frac{1}{\lambda(p)}\left(\widetilde{f}_{p}\right)_{w z_{j}}^{\prime \prime}(0) \cdot \overline{E_{l}(p)^{t}}=\frac{1}{\lambda(p)} L_{j}\left(\widetilde{f}_{w}^{\prime}\right)(p) \cdot \overline{E_{l}(p)^{t}} \\
& c_{l}(p):=\left.\frac{\partial^{2} g_{p}^{*}}{\partial z_{l} \partial w}\right|_{0}=\frac{1}{\lambda(p)}\left(g_{p}\right)_{w z_{l}}^{\prime \prime}(0)=\left.\frac{1}{\lambda(p)} L_{l}\left(g_{w}^{\prime}-2 i \widetilde{f}_{w}^{\prime} \cdot \overline{\widetilde{f}(p)}^{t}\right)\right|_{0}=\frac{2 i}{\lambda(p)} \overline{T(\widetilde{f})} \cdot L_{l}(\widetilde{f})^{t} \\
& r(p):=\left.\frac{1}{2} \operatorname{Re}\left(\frac{\partial^{2} g_{p}^{*}}{\partial w^{2}}\right)\right|_{0}=\frac{1}{2 \lambda(p)} \operatorname{Re}\left(\left(g_{p}\right)_{w w}^{\prime \prime}(0)\right)=\frac{1}{2 \lambda(p)} \operatorname{Re}\left(g_{w w}^{\prime \prime}-2 i \widetilde{f}_{w w}^{\prime \prime} \cdot \overline{\widetilde{f}(p)} t\right) .
\end{aligned}
$$

Define

$$
\begin{aligned}
G_{p}:= & \left(\frac{z^{*}-\mathbf{a}(p) w^{*}}{1+2 i\left\langle z^{*}, \overline{\mathbf{a}(p)}\right\rangle+(r(p)-i\langle\mathbf{a}(p), \overline{\mathbf{a}(p)}\rangle) w^{*}},\right. \\
& \left.\frac{w^{*}}{1+2 i\left\langle z^{*}, \overline{\mathbf{a}(p)}\right\rangle+(r(p)-i\langle\mathbf{a}(p), \overline{\mathbf{a}(p)}\rangle) w^{*}}\right) .
\end{aligned}
$$

Then $G_{p} \in \operatorname{Aut}_{0}\left(\mathbf{H}_{N}\right)$. Finally, $F_{p}^{* *}$ is the composition of $F_{p}^{*}$ with $G_{p}$ :

$$
F_{p}^{* *}:=\left(f_{p}^{* *}, \phi_{p}^{* *}, g_{p}^{* *}\right)=\left(\tilde{f}_{p}^{* *}, g_{p}^{* *}\right):=G_{p} \circ F_{p}^{*} .
$$

As in the work of [Hu1] [HJ], the following lemma is of fundamental importance for the understanding of the map $F$ :

Lemma 2.0. ([§2, Lemma 5.3, Hu1]): Let $F$ be a $C^{2}$-smooth $\mathrm{CR}$ map from a connected open subset $M \subset \mathbf{H}_{n}$ into $\mathbf{H}_{N}$. For each $p \in M, F_{p}^{* *}$, defined as above, satisfies the normalization condition:

$$
f_{p}^{* *}=z+\frac{i}{2} a_{p}^{* *(1)}(z) w+o_{w t}(3), \phi_{p}^{* *}=\phi_{p}^{* *}(2)(z)+o_{w t}(2), g_{p}^{* *}=w+o_{w t}(4)
$$

with

$$
\left\langle\bar{z}, a_{p}^{* *(1)}(z)\right\rangle|z|^{2}=\left|\phi_{p}^{* *(2)}(z)\right|^{2}
$$

In passing, we notice that there is a $\tau_{p}^{* *} \in \operatorname{Aut}_{0}\left(\mathbf{H}_{N}\right)$ such that

$$
F_{p}^{* *}=\tau_{p}^{* *} \circ F_{p}
$$

Also, for a fixed $p_{0} \in M$, we can make $C_{l}(p)$ in (2.1.3) depend smoothly on $E_{j}$ and $p$ for $p \approx p_{0}$.

From (2.2.2), it is easy to see that

$$
\begin{aligned}
& a_{p}^{* *(1)}(z)=z \mathcal{A}(p) \text { with } \mathcal{A}(p) \text { a certain } \\
& (n-1) \times(n-1) \text { semi-positive Hermitian matrix. }
\end{aligned}
$$

Moreover, it is independent of the choice of $C_{j}(p)$ by the following formula (see [pp224, (2.3), HJ]):

$$
P_{j}^{l}:=\left.\frac{\partial^{2}\left(f_{p}\right)_{l}^{* *}}{\partial z_{j} \partial w}\right|_{0}=d_{l j}(p)-a_{l}(p) c_{j}(p)-\delta_{j}^{l}\left(i|\mathbf{a}(p)|^{2}+r(p)\right), 1 \leq l, j \leq n-1
$$


Using the aforementioned formulas, one gets (see [pp231, (4.1), HJ]):

$$
\begin{aligned}
& 2 \lambda P_{l}^{j}=2 L_{l}\left({\widetilde{f_{w}}}^{\prime}\right) \cdot{\overline{L_{j}(\widetilde{f})}}^{t}-\frac{2}{\lambda}\left({\widetilde{f_{w}^{\prime}}}_{L_{j}(\widetilde{f})}{ }^{t}\right) \cdot L_{l}\left(g_{w}^{\prime}-2 i{\widetilde{f_{w}^{\prime}}}^{\prime} \cdot \overline{\widetilde{f}}^{t}\right)- \\
& -2 i \delta_{l}^{j}\left|\tilde{f}_{w}^{\prime}\right|^{2}-\delta_{l}^{j} \operatorname{Re}\left\{g_{w w}^{\prime \prime}\right\}+2 \delta_{l}^{j} \operatorname{Re}\left\{i \widetilde{f_{w w}^{\prime \prime}} \cdot \overline{\widetilde{f}}\right\}
\end{aligned}
$$

and

$q_{l}^{j}:=2 \lambda P_{l}^{j}-\delta_{l}^{j}\left(2 i T^{2}(\widetilde{f}) \cdot \overline{\widetilde{f}}^{t}-T^{2}(g)\right)=2 L_{l}\left({\widetilde{f_{w}}}^{\prime}\right) \cdot{\overline{L_{j}(\widetilde{f})}}^{t}-\frac{4 i}{\lambda(p)}\left({\widetilde{f_{w}^{\prime}}}_{L_{j}(\widetilde{f})}^{t}\right)\left(L_{l}(\widetilde{f}) \cdot{\overline{\widetilde{f_{w}^{\prime}}}}^{t}\right)$

Another way to see that $\mathcal{A}(p)$ is independent of the choice of $C_{j}$ is as follows:

In passing, we mention that there is a typo in the expressions of [(4.1)-(4.3), HJ]: The indices $\{l, j\}$ in the second term after the equality sign should be switched to $\{j, l\}$.

First, we notice that

$$
\left(\phi_{p}^{* *}\right)_{j}=\frac{\left(\phi_{p}^{*}\right)_{j}-b_{j}(p) g_{p}^{*}}{1+2 i<\widetilde{f_{p}^{*}, \overline{\mathbf{a}(p)}}>-(-r(p)+i<\mathbf{a}(p), \overline{\mathbf{a}(p)}>) g_{p}^{*}}
$$

As in [Hu1], write $\phi_{p}^{* *(2)}=\sum_{k, l=1}^{n-1} q_{k l} z_{k} z_{l}$ with $q_{k l}=q_{l k}=\left(q_{k l}^{1}, \cdots, q_{k l}^{(N-n)}\right)$. Comparing the coefficients of terms with factor $z_{l} z_{k}$ in the Taylor expansion of (2.3.4) (or see $[(2.6), \mathrm{Hu}])$, it follows easily that

$$
\begin{aligned}
& q_{k l}^{j}(p)=\frac{1}{2} \frac{\partial^{2}\left(\phi_{p}^{*}\right)_{j}}{\partial z_{l} \partial z_{k}}(0)=\frac{1}{2} \frac{\partial^{2}\left(\phi_{p}^{* *}\right)_{j}}{\partial z_{l} \partial z_{k}}(0) \\
& =\frac{1}{2 \sqrt{\lambda(p)}}\left(\widetilde{f}_{p}\right)_{z_{l} z_{k}}^{\prime \prime}(0) \cdot{\overline{C_{j}(p)}}^{t}=\frac{1}{2 \sqrt{\lambda(p)}} L_{l} L_{k}(\tilde{f})(p) \cdot{\overline{C_{j}(p)}}^{t}
\end{aligned}
$$

Hence, by (2.3.5) and (2.2.1) it follows that

$$
\begin{aligned}
& z \mathcal{A}(p) \bar{z}^{t}|z|^{2}=\left|\phi_{p}^{* *(2)}(z)\right|^{2}=\sum_{k, l, k^{\prime}, l^{\prime}=1}^{n-1}<q_{k l}, \overline{q_{k^{\prime} l^{\prime}}}>z_{k} z_{l} \overline{z_{k^{\prime}} z_{l^{\prime}}}= \\
& =\sum_{k, l, k^{\prime}, l^{\prime}=1}^{n-1} K_{k l \overline{k^{\prime} l^{\prime}}}(p) z_{k} z_{l} \overline{z_{k^{\prime}} z_{l^{\prime}}} \\
& \text { with } K_{k l \overline{k^{\prime} l^{\prime}}}(p)=\frac{1}{4 \lambda(p)}<L_{l} L_{k}(\tilde{f})(p), \overline{L_{l^{\prime}} L_{k^{\prime}}(\tilde{f})(p)}>- \\
& -\frac{1}{4 \lambda^{2}(p)} \sum_{j=1}^{n-1}\left(L_{l} L_{k}(\tilde{f})(p) \cdot \overline{E_{j}(p)} t\right)\left(\overline{L_{l^{\prime}} L_{k^{\prime}}(\widetilde{f})(p)} \cdot E_{j}(p)^{t}\right) .
\end{aligned}
$$

In particular, we conclude that $\left|\phi_{p}^{* *}(z)\right|^{2}$ and thus $\mathcal{A}(p)$ is independent of the choice of $C_{j}$. With this property at our disposal, we make the following definition:

Definition 2.1. The rank of $\mathcal{A}(p)=-2 i\left(P_{j}^{l}\right)_{1 \leq j, l \leq(n-1)}$, which we denote by $R k_{F}(p)$, is called the geometric rank of $F$ at $p$. 
Notice that $\mathcal{A}(p)$ depends continuously on $p$, and $R k_{F}(p)$ is a lower semi-continuous function on $p$.

2.2. In this subsection, we present an elementary but useful lemma based on the calculations in $\S 2.1$. Let $\sigma \in \operatorname{Aut}_{0}\left(\mathbf{H}_{n}\right)$ be given by

$$
\sigma(z, w):=\frac{\left(\lambda(z+a w) \cdot U, \lambda^{2} w\right)}{q(z, w)}
$$

with $q(z, w)=1-2 i<\bar{a}, z>+\left(r-i|a|^{2}\right) w$, where $\lambda>0, r \in \mathbf{R}, a$ is an (n-1)-tuple and $U$ is an $(n-1) \times(n-1)$ unitary matrix. Let $\tau^{*} \in \operatorname{Aut}_{0}\left(\mathbf{H}_{N}\right)$ be given by

$$
\tau^{*}\left(z^{*}, w^{*}\right):=\frac{\left(\lambda^{*}\left(z^{*}+a^{*} w^{*}\right) \cdot U^{*}, \lambda^{* 2} w^{*}\right)}{q^{*}\left(z^{*}, w^{*}\right)}
$$

with $q^{*}\left(z^{*}, w^{*}\right)=1-2 i<\overline{a^{*}}, z^{*}>+\left(r^{*}-i\left|a^{*}\right|^{2}\right) w^{*}$, where $\lambda^{*}>0, r^{*} \in \mathbf{R}, a^{*}$ is an $(N-1)$-tuple and $U^{*}$ is an $(N-1) \times(N-1)$ unitary matrix.

Lemma 2.2. (A). Suppose that $F=(f, \phi, g)$ and $F^{*}=\left(f^{*}, \phi^{*}, g^{*}\right)$ are two $C^{2}$ smooth CR map from a neighborhood of 0 in $\mathbf{H}_{n}$ into $\mathbf{H}_{N}(N \geq n>1)$, that satisfy the normalization condition (2.2.1). Assume that $F^{*}=\tau^{*} \circ F \circ \sigma$ with $\sigma, \tau^{*}$ given in (2.4.1) and (2.4.2), respectively. Then it holds that

$$
\begin{gathered}
\lambda^{*}=\lambda^{-1}, a_{1}^{*}=-\lambda^{-1} a \cdot U, a_{2}^{*}=0, r^{*}=-\lambda^{-2} r, \\
U^{*}=\left(\begin{array}{cc}
U^{-1} & 0 \\
0 & U_{22}^{*}
\end{array}\right),
\end{gathered}
$$

where $a^{*}=\left(a_{1}^{*}, a_{2}^{*}\right)$ with $a_{1}^{*}$ its first $(n-1)$ components, $U_{22}^{*}$ is an $(N-n) \times(N-n)$ unitary matrix. Conversely, suppose $\tau^{*}$ and $\sigma$, given in (2.4.1)-(2.4.2), are related by (2.5.1)-(2.5.2). And suppose that $F$ satisfies the normalization condition (2.2.1). Then $F^{*}$ must also satisfy the normalization in (2.2.1).

(B). Let $F_{1}$ be a non-constant $C^{2}$-CR map from $M \subset \mathbf{H}_{n}$ into $\mathbf{H}_{N}$. Assume that $F_{2}=\tau \circ F_{1} \circ \sigma$ with $\sigma \in \operatorname{Aut}\left(\mathbf{H}_{n}\right)$ and $\tau \in \operatorname{Aut}\left(\mathbf{H}_{N}\right)$. Then $R k_{F_{2}}(p)=R k_{F_{1}}(\sigma(p))$.

(C). Suppose that $F$ satisfies the normalization condition as in (2.2.1) and assume that $R k_{F}(0)=\kappa_{0}>0$. Then there are $\sigma \in \operatorname{Aut}_{0}\left(\mathbf{H}_{n}\right)$ and $\tau \in \operatorname{Aut}_{0}\left(\mathbf{H}_{N}\right)$ such that $\tau \circ F \circ \sigma:=(f, \phi, g)$ satisfies the following normalization condition:

$$
\begin{aligned}
& f_{j}=z_{j}+\frac{i \mu_{j}}{2} z_{j} w+o_{w t}(3), \text { for } j \leq \kappa_{0} \\
& f_{j}=z_{j}+o_{w t}(3), \text { for } j>\kappa_{0} \\
& g=w+o_{w t}(4), \\
& \phi=\phi^{(2)}(z)+o_{w t}(2), \\
& \frac{\partial^{2} f_{j}}{\partial w^{2}}(0)=0, \text { for } j \leq \kappa_{0}
\end{aligned}
$$

where $\mu_{1}=1$ and $\mu_{j} \geq 1$ for $j \leq \kappa_{0}$.

(D). Let $F$ be a $C^{2}-C R$ map from a small neighborhood of 0 in $\mathbf{H}_{n}$ into $\mathbf{H}_{N}$ satisfying the normalization in (2.5.3) with $\kappa_{0}>0$. Then for any complex line $L$ transversal to 
$\mathbf{H}_{n}$ at 0 , if $\left.F\right|_{L}$ is linear fractional, then $L$ must be contained in $S_{0}:=\left\{(z, w) \in \mathbf{C}^{n}\right.$ : $\left.z_{1}=\cdots=z_{\kappa_{0}}=0\right\}$.

Proof of Lemma 2.2. Let $F$ and $F^{*}$ be as in Lemma $2.2(\mathrm{~A})$. Write $F^{*}=\left(\widetilde{f^{*}}, g^{*}\right)=$ $\left(f^{*}, \phi^{*}, g^{*}\right)$. Then a direct computation shows that $g^{*}=\lambda^{2} \lambda^{* 2} w+O\left(|(z, w)|^{2}\right)$ and $\widetilde{f^{*}}=\lambda^{*}(\lambda(z+a w) \cdot U, 0) \cdot U^{*}+\lambda^{*} a^{*} \lambda^{2} U^{*} w+O\left(|(z, w)|^{2}\right)$. Hence, we get $\lambda^{*}=\lambda^{-1}$, $(z U, 0) U^{*}=(z, 0)$, and $(a U, 0) U^{*}+\lambda a^{*} U^{*}=0$. Write

$$
U^{*}=\left(\begin{array}{cc}
U_{11}^{*} & U_{12}^{*} \\
U_{21}^{*} & U_{22}^{*}
\end{array}\right)
$$

Then it follows that $U U_{11}^{*}=\mathrm{Id}$, or $U_{11}^{*}=U^{-1}$. Since $U^{*}$ is unitary, we see that $U_{12}^{*}=0, U_{21}^{*}=0$ and $U_{22}^{*}$ is also unitary. Hence, we have $a+\lambda a_{1}^{*} U_{11}^{*}=0$ and $a_{2}^{*} U_{22}=0$. This gives that $a_{2}^{*}=0$ and $a_{1}^{*}=-\lambda^{-1} a U$. To get the relation between $r^{*}$ and $r$, we notice that

$$
\begin{aligned}
& g^{*}(0, w)=\frac{\lambda^{* 2} g \circ \sigma(0, w)}{q^{*}(\widetilde{f} \circ \sigma(0, w), g \circ \sigma(0, w))} \\
& =\frac{w}{\left(1+\left(r-i|a|^{2}\right) w\right)\left(1-2 i<\overline{a_{1}^{*}}, f \circ \sigma(0, w)>+\left(r^{*}-i\left|a^{*}\right|^{2}\right) g \circ \sigma(0, w)\right)}+o\left(w^{2}\right) \\
& =w\left(1-\left(r-i|a|^{2}\right) w\right)\left(1-2 i|a|^{2} w-\left(r^{*} \lambda^{2}-i|a|^{2}\right) w\right)=w\left(1-\left(r+r^{*} \lambda^{2}\right) w\right)+o\left(w^{2}\right) .
\end{aligned}
$$

Therefore, we have $r^{*}=-\lambda^{-2} r$.

Write $f=z+\frac{i}{2} z \mathcal{A} w+o_{w t}(3)$ and $f^{*}=z+\frac{i}{2} z \mathcal{A}^{*} w+o_{w t}(3)$ as before. Notice that

$f^{*}=\frac{\left(\lambda^{*} f\left(\lambda(z+a w) \cdot U / q(z, w), \lambda^{2} w / q\right)+\lambda^{*} a_{1}^{*} g\left(\lambda(z+a w) \cdot U / q(z, w), \lambda^{2} w / q\right)\right) U^{-1}}{q^{*}(f \circ \sigma(z, w), g \circ \sigma(z, w))}$.

A similar calculation then shows that

$$
\begin{aligned}
& \mathcal{A}^{*}=\lambda^{2} U \mathcal{A} U^{-1} \text { and thus } \\
& R k_{F^{*}}(0)=R k_{F}(0) ; \frac{\partial^{2} f^{*}}{\partial w^{2}}(0)=i \lambda^{2} a U \mathcal{A} U^{-1}+\lambda^{3} \frac{\partial^{2} f}{\partial w^{2}}(0) U^{-1}
\end{aligned}
$$

Conversely, suppose the formulas in (2.5.1)-(2.5.2) hold, and suppose that $F$ satisfies the normalization (2.2.1). Then $F^{*}=\left(f^{*}, \phi^{*}, g^{*}\right)$ satisfies the normalization:

$$
\begin{aligned}
& f^{*}(z, w)=z+O\left(|(z, w)|^{2}\right), \phi^{*}=\phi^{(2)}(z)+o_{w t}(2) \\
& \text { and } g^{*}(z, w)=w+O\left(|(z, w)|^{2}\right), T^{2}\left(g^{*}\right)(0)=0
\end{aligned}
$$

By [Lemma 5.3, Hu1], we conclude that $F^{*}$ also satisfies the normalization (2.2.1).

To prove the statement in (B), we just notice that in the context of (B), there are $\sigma_{0} \in \operatorname{Aut}_{0}\left(\mathbf{H}_{n}\right)$ and $\tau_{0} \in \operatorname{Aut}_{0}\left(\mathbf{H}_{N}\right)$ such that $F_{2, p}^{* *}=\tau_{0} \circ F_{1, \sigma(p)}^{* *} \circ \sigma_{0}$. Hence, by Lemma 2.2 (A) (2.5.4), we conclude the statement in (B).

Next, let $F$ be as in (C). By (2.5.4), we can apparently make $\mathcal{A}=\operatorname{diag}\left(\mu_{1}, \cdots, \mu_{\kappa_{0}}, 0, \cdots, 0\right)$ with $\mu_{j} \geq \mu_{1}=1$, by choosing $\lambda, U$ suitably. To get the normalization in the last line of $(2.5 .3)$, by $(2.5 .4)$ we need only to replace $F$ 
by $\tau \circ F \circ \sigma$, where $\sigma$ and $\tau$ are as in (2.5.1)-(2.5.2), respectively, with $U=\mathrm{Id}, \lambda=1$, $r=0, U_{22}^{*}=\mathrm{Id}$, and

$$
a=\left(\frac{2 \sqrt{-1} a_{02,1}}{\mu_{1}}, \cdots, \frac{2 \sqrt{-1} a_{02, \kappa_{0}}}{\mu_{\kappa_{0}}}, 0, \cdots, 0\right) \text { where } a_{02, j}=\frac{1}{2} \frac{\partial^{2} f_{j}}{\partial w^{2}}(0)
$$

This proves (C).

Now, we turn to the proof of $(\mathrm{D})$. Let $L$ be a line defined by $z=c t=$ $\left(c_{1}, \cdots, c_{n-1}\right) t, w=t$ and assume that $\left.F\right|_{L}$ is linear fractional. Let

$$
\sigma=\frac{(z+c w, w)}{1-2 i<z, \bar{c}>-i|c|^{2} w}, \quad \tau=\frac{\left(z^{*}-(c, 0) w^{*}, w^{*}\right)}{1+2 i<z^{*}, \overline{(c, 0)}>-i|c|^{2} w^{*}}
$$

and $F^{*}=\tau \circ F \circ \sigma$. Then $F^{*}$ must map $\{(0, w): \operatorname{Im}(w) \geq 0\}$ into itself. However $f_{j}^{*}(0, t)=\frac{i}{2} \mu_{j} c_{j} t^{2}+o\left(t^{2}\right)$. We see that $c_{j}=0$ for $j \leq \kappa_{0}$. The proof of Lemma 2.2 is now complete.

Let $F$ be a $C^{2}$-smooth CR map from $M \subset \mathbf{H}_{n}$ into $\mathbf{H}_{N}$ as before. We say that $F$ is $k$-linear at $p \in M$ if there are $\sigma \in \operatorname{Aut}_{0}\left(\mathbf{H}_{n}\right)$ and $\tau \in \operatorname{Aut}_{0}\left(\mathbf{H}_{N}\right)$ such that $\tau \circ F_{p} \circ \sigma\left(z_{1}, \cdots, z_{k-1}, 0, \cdots, 0, w\right)=\left(z_{1}, \cdots, z_{k-1}, 0, \cdots, 0, w\right)$. From Lemma $2.2(\mathrm{C})$ (D), it follows easily that if $R k_{F}(p) \geq \kappa_{0}$, then $F$ cannot be $\left(n-\kappa_{0}+1\right)$-linear at $p$. The following theorem, which is regarded as the main technical Theorem of the paper, says that under a certain assumption, $F$ is always $\left(n-\kappa_{0}\right)$-linear at $p$ :

THEOREM 2.3. Let $F$ be a non-constant $C^{3}-C R$ map from a connected open subset $M$ of $\mathbf{H}_{n}$ into $\mathbf{H}_{N}$. Assume that $R k_{F}(p) \equiv \kappa_{0}<n-1$ is constant for each $p \in M$. Then $F$ is $\left(n-\kappa_{0}\right)$-linear at any point in $M$. Moreover for each $p \in M$, there is a unique affine complex subspace $S_{p}$ of dimension $\left(n-\kappa_{0}\right)$ transversal to $\mathbf{H}_{n}$ such that the restriction of $F$ to $S_{p}$ is linear. Also, $S_{p}-p$, as an element in the complex Grassmannian manifold $G_{n, n-\kappa_{0}}(\mathbf{C})$ of $\left(n-\kappa_{0}\right)$-dimensional linear subspaces in $\mathbf{C}^{n}$, depends $C^{1}$-continuously on $p$.

3. Analysis of the associated differential equations. Let $F$ be a CR map from a small neighborhood $M$ of 0 in $\mathbf{H}_{n}$ into $\mathbf{H}_{N}(N \geq n>1)$ with $F(0)=0$. We will prove in this section and $\S 4$ that it must be $\left(n-\kappa_{0}\right)$-linear at each point in $M$ if it is $C^{3}$-smooth and $R k_{F} \equiv \kappa_{0}<n-1$. By [Theorem 4.2, Hu1], we can assume, in what follows, that $\kappa_{0}>0$. By the Lewy extension theorem ([BER]), $F$ extends holomorphically to a subdomain $\Omega$ in $\mathbf{S}_{n}$ which has $M$ as part of its smooth boundary. In this section, $F$ is only assumed to be $C^{2}$-smooth over $M$.

3.1. In this subsection, we derive a system of differential equations by (2.2.2), through which we take control of $F$. Then we derive some consequences of the system for the purpose of the later application.

For each $p \in M$, write $\mathcal{E}_{p}:=\left\{\xi(p)=\left(\xi_{1}(p), \cdots, \xi_{n-1}(p)\right) \in \mathbf{C}^{n-1}: \quad \xi(p) \cdot \mathcal{A}_{p}\right.$. $\left.\overline{\xi(p)}^{t}=0\right\}$. Since $\mathcal{A}_{p}$ is semi-positive, $\mathcal{E}_{p}=\left\{\xi(p): \quad \xi(p) \cdot \mathcal{A}_{p}=0\right\}$. By $(2.2 .2)$, it follows that $\xi(p) \in \mathcal{E}_{p}$ if and only if $\phi_{p}^{* *(2)}(\xi(p))=0$. We start with the following generalization of [Lemma 2.1, Lemma 4.3] of [Hu1]: 
LEMMA 3.1. $\xi(p)=\left(\xi_{1}(p), \cdots, \xi_{n-1}(p)\right) \in \mathcal{E}_{p}$ if and only if

$$
\begin{aligned}
& \sum_{j, l=1}^{n-1} \xi_{j}(p) \xi_{l}(p) L_{j} L_{l}(\widetilde{f})(p)=\sum_{l=1}^{n-1} \Lambda_{l}(p) L_{l}(\tilde{f})(p) \text { with } \\
& \Lambda_{l}=\sum_{j=1}^{n-1} \frac{4 \sqrt{-1}}{\lambda(p)} \xi_{j}(p) \xi_{l}(p) \overline{\widetilde{f_{w}^{\prime}}(p)} \cdot L_{j}(\widetilde{f})^{t}(p) .
\end{aligned}
$$

Proof of Lemma 3.1. The proof can be done in an essentially identical way as for the proof of [Lemma 2.1, Hu1], with a slight modification due to the fact that we now only know that $\phi_{p}^{* *(2)}(\xi(p))=0$. For convenience of the reader, we include here the following details:

Comparing the coefficients of terms of the form $z_{l} z_{k}$ in the Taylor expansion of (2.3.4) and using the given hypothesis, it follows easily that $\sum_{k, l=1}^{n-1} \xi_{l}(p) \xi_{k}(p) q_{k l}^{j}(p)=$ 0 for any $j$. By (2.3.5), one gets that the $(N-1)$-tuple $\sum_{k, l} \xi_{k} \xi_{l}\left(\widetilde{f}_{p}\right)_{z_{l} z_{k}}^{\prime \prime}(0)=$ $\sum_{k, l=1}^{n-1} \xi_{k} \xi_{l} L_{l} L_{k}(\widetilde{f})$ stays in the space spanned by $\left\{E_{1}, \cdots, E_{n-1}\right\}$. Namely, there exist scalar numbers $\left\{\lambda_{j}^{*}(p)\right\}$ such that

$$
\sum_{k, l=1}^{n-1} \xi_{k} \xi_{l} L_{l} L_{k}(\widetilde{f})(p)=\sum_{j=1}^{n-1} \lambda_{j}^{*}(p) E_{j}(p)
$$

Next, considering the Taylor expansion of $\left(f_{p}^{* *}\right)_{j}$ in the following

$$
\left(f_{p}^{* *}\right)_{j}=\frac{\left(f_{p}^{*}\right)_{j}-a_{j}(p) g_{p}^{*}}{1+2 i<\widetilde{f_{p}^{*}}, \overline{\mathbf{a}(p)}>-(-r(p)+i<\mathbf{a}(p), \overline{\mathbf{a}(p)}>) g_{p}^{*}}
$$

and using (2.2.1), we obtain $\sum e_{k l}^{j}(p) z_{k} z_{l}-2 i \sum_{l} \overline{a_{l}}(p) z_{j} z_{l} \equiv 0$, where $e_{k l}^{j}(p)=$ $\frac{1}{2} \frac{\partial^{2}\left(f_{p}^{*}\right)_{j}}{\partial z_{l} \partial z_{k}}(0)$. Hence, it follows that $e_{k l}^{j}(p)=\sqrt{-1}\left(\delta_{k}^{j} \overline{a_{l}}(p)+\delta_{l}^{j} \overline{a_{k}}(p)\right)$. Therefore,

$$
\frac{1}{2 \lambda} L_{l} L_{k}(\tilde{f})(p){\overline{E_{j}}}^{t}=\frac{\sqrt{-1} \delta_{k}^{j}}{\lambda(p)} \overline{E_{w}}(p) \cdot E_{l}(p)^{t}+\frac{\sqrt{-1} \delta_{l}^{j}}{\lambda(p)} \overline{E_{w}}(p) \cdot E_{k}(p)^{t}
$$

Combing this with (3.1.1) and making use of the orthogonality: $E_{l}(p) \cdot{\overline{E_{j}(p)}}^{t}=\lambda(p) \delta_{l}^{j}$, we get $\lambda_{j}^{*}=\frac{2 \sqrt{-1}}{\lambda} \sum_{k, l=1}^{n-1} \xi_{k}(p) \xi_{l}(p)\left(\delta_{k}^{j} \overline{E_{w}(p)} \cdot E_{l}(p)^{t}+\delta_{l}^{j} \overline{E_{w}(p)} \cdot E_{k}(p)^{t}\right)$. Returning to (3.1.1), we get the proof of Lemma 3.1.

Denote by $\mathcal{S}_{0}=\left\{(j, l): 1 \leq j \leq \kappa_{0}, 1 \leq l \leq(n-1), j \leq l\right\}$ and write $\mathcal{S}:=\{(j, l):$ $(j, l) \in \mathcal{S}_{0}$, or $\left.j=\kappa_{0}+1, l \in\left\{\kappa_{0}+1, \cdots, N-n-\frac{\left(2 n-\kappa_{0}-1\right) \kappa_{0}}{2}\right\}\right\}$.

Lemma 3.2. Let $F$ be a $C^{2}$-smooth $C R$ map from $M \subset \mathbf{H}_{n}$ into $\mathbf{H}_{N}$ with $F(0)=0$ and $R k_{F}(0)=\kappa_{0}$. Let $P\left(n, \kappa_{0}\right)=\frac{\kappa_{0}\left(2 n-\kappa_{0}-1\right)}{2}$. Then $N \geq n+P\left(n, \kappa_{0}\right)$ and there are $\sigma \in \operatorname{Aut}_{0}\left(\mathbf{H}_{n}\right)$ and $\tau \in \operatorname{Aut}_{0}\left(\mathbf{H}_{N}\right)$ such that $\tau \circ F \circ \sigma:=(f, \phi, g)$ satisfies the following normalization condition: 


$$
\begin{aligned}
& f_{j}=z_{j}+\frac{i \mu_{j}}{2} z_{j} w+o_{w t}(3), \quad \frac{\partial^{2} f_{j}}{\partial w^{2}}(0)=0, j=1 \cdots, \kappa_{0}, \mu_{j}>0, \\
& f_{j}=z_{j}+o_{w t}(3), \quad j=\kappa_{0}+1, \cdots, n-1 \\
& g=w+o_{w t}(4) \\
& \phi_{j l}=\mu_{j l} z_{j} z_{l}+o_{w t}(2), \\
& \text { where }(j, l) \in \mathcal{S} \text { with } \mu_{j l}>0 \text { for }(j, l) \in \mathcal{S}_{0} \text { and } \mu_{j l}=0 \text { otherwise. }
\end{aligned}
$$

Moreover, $\mu_{j l}=\sqrt{\mu_{j}+\mu_{l}}$ for $j, l \leq \kappa_{0} j \neq l$; and $\mu_{j l}=\sqrt{\mu_{j}}$ if $j \leq \kappa_{0}$ and $l>\kappa_{0}$ or if $j=l \leq \kappa_{0}$. Here and in what follows, we label the components of $\phi$ by double indices $(j, l) \in \mathcal{S}$.

Proof of Lemma 3.2. By Lemma 2.2, we can assume that $F$ already takes the form (2.5.3). From the equation (2.2.2), we get $\sum_{j=1}^{\kappa_{0}} \mu_{j}\left|z_{j}\right|^{2}|z|^{2}=\sum_{l}\left|\phi_{l}^{(2)}(z)\right|^{2}$. Write $\phi_{j}^{(2)}(z)=\sum_{k \leq l} a_{k l}^{j} z_{k} z_{l}$. We then have

$$
\sum_{j=1}^{\kappa_{0}} \mu_{j}\left|z_{j}\right|^{2}|z|^{2}=\sum_{j} a_{k l}^{j} a_{k^{\prime} l^{\prime}}^{j} z_{k} z_{l} \overline{z_{k^{\prime}} z_{l^{\prime}}}
$$

Write $\alpha_{j l}=\left(a_{j l}^{1}, \cdots, a_{j l}^{N-n}\right)$. We have

$\sum_{j=1}^{\kappa_{0}} \mu_{j}\left|z_{j}\right|^{2}|z|^{2}=\sum_{k \leq l, k^{\prime} \leq l^{\prime}}\left(\alpha_{k l}, \overline{\alpha_{k^{\prime} l^{\prime}}}\right) z_{k} z_{l} \overline{z_{k^{\prime}} z_{l^{\prime}}}$. We immediately see that $\alpha_{k l} \perp$ $\alpha_{k^{\prime} l^{\prime}}$ for $(k, l) \neq\left(k^{\prime}, l^{\prime}\right)$, and $\left|\alpha_{k l}\right|^{2}=\mu_{k}+\mu_{l}$ for $k, l \leq \kappa_{0}, k \neq l ;\left|\alpha_{k l}\right|^{2}=\mu_{k}$ for $k \leq \kappa_{0}, l>\kappa_{0}$ or $k=l \leq \kappa_{0}$. Hence, $\left\{\alpha_{k l}\right\}_{(k, l) \in \mathcal{S}_{0}}$ is a linearly independent system, which implies that $N-n \geq\left|\mathcal{S}_{0}\right|=P\left(n, \kappa_{0}\right)$. Next, extend $\left\{\frac{\alpha_{j l}}{\left|\alpha_{j l}\right|}\right\}_{(j, l) \in \mathcal{S}_{0}}$ to an $(N-n) \times(N-n)$ unitary matrix $\widetilde{U}$ and replace $\phi$ by $\phi \cdot \overline{\widetilde{U}}^{t}$. Then the rest statements in Lemma 3.2 can be easily seen.

We remark that in Lemma 3.2, we can further make $\mu_{j} \geq \mu_{1}=1$ if we like. But we do not require such a normalization for the convenience of later application.

For the rest of this subsection, we fix an integer $k$ with $\kappa_{0}+1 \leq k \leq(n-1)$, and let $I_{k}(p)=\left(\xi_{1}, \cdots, \xi_{n-1}\right) \in \mathcal{E}_{p}$ be such that its $j^{\text {th }}$ component is $\delta_{j}^{k}$ for $j>$ $\kappa_{0}$. Apparently, under the assumption that $R k_{F}(p) \equiv \kappa_{0}$ is constant, $I_{k}(p)$ depends continuously on $p$ and is uniquely determined by the linear equations:

$$
\sum_{j=1}^{\kappa_{0}} P_{j}^{l} \xi_{j}=-P_{k}^{l}, \text { for } l=1, \cdots, \kappa_{0} .
$$

The following lemma will be basic for our later discussion:

LEMMA 3.3. Assume that $F$ is normalized as in (3.2), and assume that $R k_{F}(p) \equiv$ $\kappa_{0}$ for $p \approx 0$ with $0<\kappa_{0}<n-1$.

(A): For any $j, l, l^{\prime} \in\{1, \cdots, n-1\}$, it holds that

$$
L_{j}\left(I_{k}\right), \overline{L_{j}}\left(I_{k}\right), \quad L_{j}\left(\Lambda_{l}\right), \overline{L_{j} L_{l}}\left(I_{k}\right), \overline{L_{j} L_{l}}\left(\Lambda_{l^{\prime}}\right) \in C^{0}(M) .
$$


Moreover, write $I_{k}(p)=\left(\xi_{1}(p), \cdots, \xi_{\kappa_{0}}(p), 0, \cdots, 1, \cdots, 0\right)$ as above. Then for $j \leq$ $\kappa_{0}, k^{\prime}>\kappa_{0}$,

$$
\begin{aligned}
& \xi_{j}=\frac{2 i}{\mu_{j}} \sum_{l=j+1}^{n-1} \frac{\partial^{2} \phi_{j l}}{\partial z_{k} \partial w}(0) \overline{\mu_{j l} z_{l}}+\frac{2 i}{\mu_{j}} \sum_{j^{\prime}<j} \frac{\partial^{2} \phi_{j^{\prime} j}}{\partial z_{k} \partial w}(0) \overline{\mu_{j^{\prime} j} z_{j^{\prime}}}+ \\
& +\frac{4 i}{\mu_{j}} \frac{\partial^{2} \phi_{j j}}{\partial z_{k} \partial w}(0) \overline{\mu_{j j} z_{j}}+\frac{2 i}{\mu_{j}} L_{k} T\left(f_{j}\right)+o_{w t}(1) \\
& \overline{L_{k^{\prime}}}\left(\xi_{j}\right)=\frac{2 i}{\mu_{j}} \frac{\partial^{2} \phi_{j k^{\prime}}}{\partial z_{k} \partial w}(0) \overline{\mu_{j k^{\prime}}}+o_{w t}(0) .
\end{aligned}
$$

(B): With the notation in (A), it holds that

$$
\begin{aligned}
& \sum_{j, l=1}^{n-1} \overline{L_{k}}\left(\xi_{j} \xi_{l}\right) L_{j} L_{l}(\tilde{f})+4 i \sum_{l=1}^{n-1} \xi_{l} L_{l} T(\tilde{f})=\sum_{l=1}^{n-1} \overline{L_{k}}\left(\Lambda_{l}\right) L_{l}(\tilde{f})+2 i \Lambda_{k} T(\tilde{f}) \\
& \quad \sum_{j, l=1}^{n-1}{\overline{L_{k}}}^{2}\left(\xi_{j} \xi_{l}\right) L_{j} L_{l}(\tilde{f})+8 i \sum_{l=1}^{n-1} \overline{L_{k}}\left(\xi_{l}\right) L_{l} T(\tilde{f})-8 T^{2}(\tilde{f}) \\
& =\sum_{l=1}^{n-1}{\overline{L_{k}}}^{2}\left(\Lambda_{l}\right) L_{l} \tilde{f}+4 i \overline{L_{k}}\left(\Lambda_{k}\right) T(\tilde{f})
\end{aligned}
$$

(C): For any $(j, l) \in \mathcal{S}$, it holds that $\frac{\partial^{2} \phi_{j l}}{\partial z_{k} \partial w}(0)=0$ and $\frac{\partial^{2} f}{\partial w^{2}}(0)=0$. Also, for $(j, l) \in \mathcal{S}$ with $l \neq k$, it holds that $\frac{\partial^{2} \phi_{j l}}{\partial w^{2}}(0)=0$. When $l=k, \frac{\partial^{2} \phi_{j k}}{\partial w^{2}}(0)=\frac{1}{4}{\overline{L_{k}}}^{2}\left(\xi_{j}\right)(0)$.

(D): It holds that

$$
\begin{aligned}
& \sum_{j, l=1}^{n-1}{\overline{L_{k}}}^{2}\left(\xi_{j} \xi_{l}\right) L_{j} L_{l}(g)+8 i \sum_{l=1}^{n-1} \overline{L_{k}}\left(\xi_{l}\right) L_{l} T(g)-8 T^{2}(g) \\
& =\sum_{l=1}^{n-1}{\overline{L_{k}}}^{2}\left(\Lambda_{l}\right) L_{l} g+4 i \overline{L_{k}}\left(\Lambda_{k}\right) T(g) .
\end{aligned}
$$

Proof of Lemma 3.3. (A): From (3.3.1), it follows that for $j \leq \kappa_{0}$

$$
\left(\xi_{1}, \cdots, \xi_{\kappa_{0}}\right)=-\left(P_{k}^{1}, \cdots, P_{k}^{\kappa_{0}}\right) P^{-1} \text { and thus } \xi_{j}=\frac{2 i}{\mu_{j}} P_{k}^{j}+o\left(\sum_{l=1}^{\kappa_{0}}\left|P_{k}^{l}\right|\right)
$$

where $P=\left(P_{l}^{j}\right)_{1 \leq j, l \leq \kappa_{0}}$ with $P(0)=\operatorname{diag}\left(\frac{i \mu_{1}}{2}, \cdots, \frac{i \mu_{\kappa_{0}}}{2}\right)$. Hence, shrinking $M$ if necessary, we see that for each $j_{0}$, there is a holomorphic function $H_{j_{0}}$ in its argument such that $\xi_{j_{0}}=H_{j_{0}}\left(P_{l}^{j}\right)$. Without loss of generality, we assume that $M$ is sufficiently small. We first prove the following:

Claim 3.4. Let $G$ be a $C^{\nu}$-smooth CR map from a neighborhood of $M$ in $\mathbf{H}_{n}$ into $\mathbf{C}^{N}$. Let $h(G)=H\left(p, \bar{p}, L^{\alpha} \overline{L^{\beta}} T \gamma(G)\right)_{|\alpha|+|\beta|+|\gamma| \leq \nu}$ with $H$ holomorphic in its argument. Let $D_{0}=L^{\alpha_{1}} \overline{L^{\beta_{1}}} T^{\gamma_{1}}$ be a differential operator along $M$. Suppose 
that there is a certain holomorphic function $H_{0}$ in its argument such that for each polynomial map $G^{*}$ from $\mathbf{C}^{n}$ into $\mathbf{C}^{N}$,

$$
D_{0}\left(h\left(G^{*}\right)\right)=H_{0}\left(p, \bar{p}, L^{\alpha} \overline{L^{\beta}} T \gamma\left(G^{*}\right)\right)_{|\alpha|+|\beta|+|\gamma| \leq \nu}
$$

Then the distribution $D_{0}(h(G))$, acting on $C_{0}^{\infty}(M)$, coincides with the continuous function $H_{0}\left(p, \bar{p}, L^{\alpha} \overline{L^{\beta}} T^{\gamma}(G)\right)_{|\alpha|+|\beta|+|\gamma| \leq \nu}$.

Proof of Claim 3.4. The claim is an immediate application of the Baouendi-Treves approximation theorem [BT]. (See already a version of this in [Lemma 4.1, Hu1]). In details, by [BT], there is a sequence of holomorphic polynomial maps, denoted by $\left\{G_{m}\right\}_{m=1}^{\infty}$, which converges to $G$ in the $C^{\nu}$-norm over $\bar{M}$. Hence $h\left(G_{m}\right) \rightarrow h(G)$ in the $C^{0}$-norm over $\bar{M}$, and $D_{0}\left(h\left(G_{m}\right)\right) \rightarrow D_{0}(h(G))$ in the sense of distribution. By the assumption, $D_{0}\left(h\left(G_{m}\right)\right)$ converges also to $H_{0}\left(p, \bar{p}, L^{\alpha} \overline{L^{\beta}} T^{\gamma}(G)\right)_{|\alpha|+|\beta|+|\gamma| \leq \nu}$ in the $C^{0}$-norm over $\bar{M}$. This completes the proof.

We continue our proof of Lemma 3.3. Recall that

$$
2 \lambda P_{l}^{j}(F)=2 L_{l}\left(\widetilde{f}_{w}^{\prime}\right) \overline{L_{j}(\widetilde{f})^{t}}-\frac{4 i}{\lambda}\left(\left(\widetilde{f}_{w}^{\prime}\right) \cdot \overline{\left.L_{j}(\widetilde{f})^{t}\right)}\right)\left(L_{l}(\widetilde{f}) \cdot{\widetilde{\widetilde{f}_{w}^{\prime}}}^{t}\right)-\delta_{l}^{j}\left(g_{w w}^{\prime \prime}-2 i \widetilde{f}_{w w}^{\prime \prime} \cdot \overline{\widetilde{f}}^{t}\right)
$$

If we replace $F$ by a polynomial map $G$ in the above formula, a direct computation then shows that $\overline{L_{l_{1}}}\left(P_{l}^{j}(G)\right)$ can be written as $G^{*}\left(p, \bar{p}, L^{\alpha} \overline{L^{\beta}} T^{\gamma}(G)\right)_{|\alpha|+|\beta|+|\gamma| \leq 2}$ with $G^{*}$ holomorphic in its argument. Hence, by Claim $3.4, \overline{L_{l_{1}}}\left(\xi_{j}\right)$ is continuous. Since $\mathcal{A}$ is Hermitian, $P_{j}^{l}=-\overline{P_{l}^{j}}$, from which it follows easily that $L_{l_{1}}\left(\xi_{j}\right) \in C^{0}(M)$. To see $\overline{L_{l_{1}} L_{l_{2}}}\left(\xi_{j}\right) \in C^{0}(M)$, we notice that by considering the $\phi_{j k}$-components of $\tilde{f}$ in (3.1.0) with $(j, l) \in \mathcal{S}_{0}$, we have for each $j \leq \kappa_{0}$ that $\xi_{j}(F)=H_{j}^{*}\left(L_{l} \widetilde{f}, L_{l_{1}} L_{l_{2}}(\widetilde{f}), \lambda(p), \widetilde{T(\widetilde{f})}\right.$. $\left.L_{l_{3}}(\widetilde{f})^{t}\right)$, where $H_{j}^{*}$ is a certain holomorphic function in its variables. Notice that

$$
\begin{aligned}
& \overline{T(\widetilde{f})} \cdot L_{j}(\tilde{f})^{t}=\frac{1}{2 i} L_{j} T(g)-\left(L_{j} T(\tilde{f})\right) \cdot \overline{\widetilde{f}} \\
& \overline{L_{l_{1}} L_{l_{2}}}\left(\frac{1}{2 i} L_{j} T(\tilde{f})-\left(L_{j} T(\tilde{f})\right) \cdot \overline{\widetilde{f}}\right) \\
& =-2 i \delta_{j}^{l_{1}} T^{2}(\tilde{f}) \cdot \overline{L_{l_{2}}(\tilde{f})}-2 i \delta_{j}^{l_{2}} T^{2}(\tilde{f}) \cdot \overline{L_{l_{1}}(\tilde{f})}-L_{j} T(\tilde{f}) \cdot \overline{L_{l_{1}} L_{l_{2}}(\widetilde{f})}
\end{aligned}
$$

(if $F$ is smooth). We easily conclude that if we replace $F$ by a polynomial map $G$ above, we have $\overline{L_{l_{1}} L_{l_{2}}}\left(\xi_{j}(G)\right)=H_{j}^{* *}\left(p, \bar{p}, L^{\alpha} \overline{L^{\beta}} T^{\gamma}(G)\right)_{|\alpha|+|\beta|+|\gamma| \leq 2}$. Hence, we see that $\overline{L_{l_{1}} L_{l_{2}}}\left(\xi_{j}\right)$ is continuous over $M$. Similarly, making use of (3.4.5) and the second formula in (3.1.0):

$$
\Lambda_{l}=\sum \frac{4 i}{\lambda} \xi_{j}\left(P_{l^{\prime}}^{j^{\prime}}\right) \xi_{l}\left(P_{l^{\prime}}^{j^{\prime}}\right) \overline{T(\widetilde{f})} \cdot L_{j}(\widetilde{f})^{t}
$$

we can show that $\overline{L_{j}}\left(\Lambda_{l}\right), L_{j}\left(\Lambda_{l}\right)$ and $\overline{L_{j^{\prime}} L_{j}}\left(\Lambda_{l}\right)$ are continuous over $M$. For the later application, we give here the following explicit formulas: 


$$
\begin{aligned}
& \overline{L_{k}}\left(\Lambda_{l}\right)=I I_{1}+I I_{2}+I I_{3}+I I_{4}, \quad \text { where } \\
& \left.I I_{1}=\sum_{j=1}^{n-1} \frac{-4 i}{\lambda^{2}} \overline{L_{k}}(\lambda)\left(\overline{\widetilde{f}_{w}^{\prime}} \cdot L_{j} \widetilde{f}^{t}\right) \xi_{j} \xi_{l}, \quad I I_{2}=\sum_{j=1}^{n-1} \frac{4 i}{\lambda} \overline{\left(L_{k}\left(\widetilde{f}_{w}^{\prime}\right)\right.} \cdot L_{j} \widetilde{f}^{t}\right) \xi_{j} \xi_{l}, \\
& I I_{3}=\frac{-8}{\lambda}\left(\widetilde{\widetilde{f}_{w}^{\prime}} \cdot\left(\widetilde{f_{w}^{\prime}}\right)^{t}\right) \xi_{l}, \quad I I_{4}=\sum_{j=1}^{n-1} \frac{4 i}{\lambda}\left(\widetilde{\widetilde{f}_{w}^{\prime}} \cdot L_{j} \widetilde{f}^{t}\right) \overline{L_{k}}\left(\xi_{j} \xi_{l}\right) \\
& {\overline{L_{k}}}^{2}\left(\Lambda_{l}\right)=\sum_{j=1}^{n-1} 4 i{\overline{L_{k}}}^{2}\left(\frac{1}{\lambda(p)}\right) K_{j} \xi_{j} \xi_{l}+\sum_{j=1}^{n-1} \overline{L_{k}}\left(\frac{8 i}{\lambda(p)}\right) \overline{L_{k}}\left(K_{j}\right) \xi_{j} \xi_{l} \\
& +\sum_{j=1}^{n-1} \overline{L_{k}}\left(\frac{8 i}{\lambda(p)}\right) K_{j} \overline{L_{k}}\left(\xi_{j} \xi_{l}\right)+\sum_{j=1}^{n-1} \frac{4 i}{\lambda} \overline{L_{k}^{2}}\left(K_{j}\right) \xi_{j} \xi_{l} \\
& +\sum_{j=1}^{n-1} \frac{8 i}{\lambda} \overline{L_{k}}\left(K_{j}\right) \overline{L_{k}}\left(\xi_{j} \xi_{l}\right)+\sum_{j=1}^{n-1} \frac{4 i}{\lambda} K_{j} \overline{L_{k}^{2}}\left(\xi_{j} \xi_{l}\right), \text { with } \\
& K_{j}=\overline{T(\widetilde{f})} \cdot L_{j}(\widetilde{f})^{t}, \quad \overline{L_{k}}\left(K_{j}\right)=\overline{L_{k} T(\widetilde{f})} \cdot L_{j}(\widetilde{f})^{t}+2 i \delta_{j}^{k} \overline{T(\widetilde{f})} \cdot T(\widetilde{f})^{t}, \\
& {\overline{L_{k}}}^{2}\left(K_{j}\right)=-4 i \delta_{j}^{k} T^{2}(\tilde{f}) \cdot{\overline{L_{k}(\widetilde{f})}}^{t}-L_{j} T(\widetilde{f}) \cdot{\overline{L_{k}^{2}(\widetilde{f})}}^{t} \text {. }
\end{aligned}
$$

Next, we have for $j \leq \kappa_{0}$ that

$$
\begin{aligned}
& 2 \lambda P_{k}^{j}=2 L_{k}\left(\widetilde{f_{w}^{\prime}}\right) \cdot \overline{L_{j}(\widetilde{f})^{t}}-\frac{4 i}{\lambda}\left(\left(\widetilde{f}_{w}^{\prime}\right) \cdot \overline{\left.L_{j}(\widetilde{f})^{t}\right)}\right)\left(L_{k}(\tilde{f}) \cdot{\overline{\widetilde{f}_{w}^{\prime}}}^{t}\right)=2 \sum_{l=j+1}^{n-1} \frac{\partial^{2} \phi_{j l}}{\partial z_{k} \partial w}(0) \overline{\mu_{j l} z_{l}}+ \\
& +4 \frac{\partial^{2} \phi_{j j}}{\partial z_{k} \partial w}(0) \overline{\mu_{j j} z_{j}}+2 \sum_{j^{\prime}<j} \frac{\partial^{2} \phi_{j^{\prime} j}}{\partial z_{k} \partial w}(0) \overline{\mu_{j^{\prime} j} z_{j^{\prime}}}+2 L_{k} T\left(f_{j}\right)+o_{w t}(1)
\end{aligned}
$$

With (3.4.3), this gives immediately (3.3.2).

To see (B), we just need to apply $\overline{L_{k}}$ and ${\overline{L_{k}}}^{2}$ to (3.1.0), respectively, with an application of Claim 3.4.

Notice that $\xi_{j}=o_{w t}(0)$ for $j \neq k$ and $\Lambda_{l}=o_{w t}(0)$. For a $(j, l) \notin \mathcal{S}_{0}$, considering the $\phi_{j l}$-component of $\tilde{f}$ in (3.4.1), and then collecting terms of weighted degree 0 , we see that $\frac{\partial^{2} \phi_{j l}}{\partial w \partial z_{k}}(0)=0$. For $(j, l) \in \mathcal{S}_{0}$, considering the $\phi_{j l}$-component in (3.4.1) we have $\left(2-\delta_{j}^{l}\right) \overline{L_{k}}\left(\xi_{j} \xi_{l}\right) L_{j} L_{l}\left(\phi_{j l}\right)(0)+4 i L_{k} T\left(\phi_{j l}\right)(0)=0$. Assume that $l \neq k$. Since $\overline{L_{k}}\left(\xi_{j} \xi_{l}\right)(0)=0$, we derive that $L_{k} T\left(\phi_{j l}\right)(0)=0$. When $l=k$, by $(3.3 .2)$, we have

$$
\begin{aligned}
0 & =\overline{L_{k}}\left(\xi_{j}\right) L_{j} L_{k}\left(\phi_{j l}\right)(0)+4 i L_{k} T\left(\phi_{j l}\right)(0)=\frac{2 i}{\mu_{j}} \mu_{j k}^{2} L_{k} T\left(\phi_{j k}\right)(0)+4 i L_{k} T\left(\phi_{j k}\right)(0)= \\
& =6 i L_{k} T\left(\phi_{j k}\right)(0) .
\end{aligned}
$$

It thus follows that $L_{k} T\left(\phi_{j k}\right)(0)=0$. This proves that $\frac{\partial^{2} \phi_{j l}}{\partial w \partial z_{k}}(0)=0$ for any $(j, l) \in \mathcal{S}$. Also, it implies by (3.3.2) that

$$
\overline{L_{k^{\prime}}}\left(\xi_{j}\right)(0)=0, \text { for } k^{\prime}>\kappa_{0}
$$


Next, we proceed to show that $T^{2}(f)(0)=0$. By the normalization condition, we need to show that $T^{2}\left(f_{j}\right)(0)=0$ for $j>\kappa_{0}$. By (3.5.2), we have ${\overline{L_{k}}}^{2}\left(\Lambda_{l}\right)(0)=$ $16 \delta_{l}^{k} T^{2}\left(f_{k}\right)(0)$. Applying $(3.4 .1)^{\prime}$, we arrive at $-8 T^{2}\left(f_{j}\right)(0)=16 \delta_{j}^{k} T^{2}\left(f_{k}\right)(0)$, from which it follows easily that $T^{2}\left(f_{j}\right)=0$. Moreover, considering the $\phi_{j l}$ component with $(j, l) \notin \mathcal{S}_{0}$ in $(3.4 .1)$, we get $T^{2}\left(\phi_{j l}\right)(0)=0$. When $(j, l) \in \mathcal{S}_{0}$, we get ${\overline{L_{k}}}^{2}\left(\xi_{j} \xi_{l}\right)(0) L_{j} L_{l}\left(\phi_{j l}\right)(0)-4 T^{2}\left(\phi_{j l}\right)(0)=0$. Hence, if $l \neq k$, since ${\overline{L_{k}}}^{2}\left(\xi_{j} \xi_{l}\right)(0)=0$, it holds that $T^{2}\left(\phi_{j l}\right)(0)=0$. If $l=k$, we get $T^{2}\left(\phi_{j k}\right)(0)=\frac{1}{4}{\overline{L_{k}}}^{2}\left(\xi_{j}\right)(0)$.

For the proof of (D), we just need to dot-multiply both sides of (3.1.0) by the vector $2 \sqrt{-1} \overline{\widetilde{f}}$ and then apply $\overline{L_{k}^{2}}$ as before.

3.2. In this subsection, we first derive a new system of differential equations by making use of Lemma 3.3. Then we use it, together with Lemma 3.3, to achieve an approximate partial linearity theorem for $F$. We also discuss the distribution introduced by the partial linearity of $F$ and its relation with $\mathcal{E}_{p}$ introduced at the beginning of $\S 3.1$.

From Lemma $3.3(\mathrm{C})$, for $k>\kappa_{0}$, we have $\frac{\partial^{2} \widetilde{f}}{\partial z_{k} \partial w}(0)=0$ for any $F$ satisfying the normalization in (3.2). This fact can be easily applied to $F_{p}^{* *}$ to derive a new system of some useful differential equations. In details, we explain it as follows:

Let $I_{k}(p)=\left(\xi_{1}, \cdots, \xi_{n-1}\right)$ and $f_{p}^{* *}=z+\frac{i}{2} w z \mathcal{A}(p)+o_{w t}(3)$ be as before. We notice that $\left\{I_{\kappa_{0}+1}(p), \cdots, I_{n-1}(p)\right\}$ is a basis of the 0 -eigenspace of the Hermitian matrix $\frac{i}{2} \mathcal{A}(p)$. By using the Schmit normalization procedure, we can find a unitary matrix $U_{(p, k)}$, whose $k$ th row is $\frac{I_{k}}{\left|I_{k}\right|}$ such that

$$
U_{(p, k)} \mathcal{A}(p) U_{(p, k)}^{-1}=\operatorname{diag}\left(\mu_{1}(p), \cdots, \mu_{\kappa_{0}}(p), 0, \cdots, 0\right) .
$$

Moreover, we can assume that the last $\left(n-\kappa_{0}-1\right)$ rows of $U_{(p, k)}$ depend continuously on $p(\approx 0)$; and at $p=0$, they are precisely $\left\{I_{\kappa_{0}+1}(0), \cdots, I_{n-1}(0)\right\}$.

Notice that $\frac{I_{k}(p)}{\left|I_{k}(p)\right|}=e_{k}^{\prime} U_{(p, k)}$, where $e_{k}^{\prime}$ is the vector in $\mathbf{C}^{n-1}$ whose component at the $j$ th position is $\delta_{j}^{k}$. Also, from the proof of Lemma 3.2, there is an $(N-n) \times$ $(N-n)$ unitary matrix $\widetilde{U_{p}}$ such that $\phi_{p}^{* *}\left(z U_{(p, k)}, w\right) \cdot{\widetilde{U_{p}}}^{-1}$ takes the form as in the last expression of (3.2). Write

$\hat{F}_{p}^{* *}=\left(f_{p}^{* *}\left(z U_{(p, k)}, w\right) U_{(p, k)}^{-1}, \phi_{p}^{* *}\left(z U_{(p, k)}, w\right){\widetilde{U_{p}}}^{-1}, g\left(z U_{(p, k)}, w\right)\right), F_{p}^{* * *}=\hat{\tau}_{p} \circ \hat{F}_{p}^{* *} \circ \hat{\sigma}_{p}$

with

$$
\hat{\sigma}_{p}=\frac{(z+\hat{a} w, w)}{1-2 i<\overline{\hat{a}}, z>-i|\hat{a}|^{2} w}, \quad \hat{\tau}_{p}=\frac{\left(z^{*}-(\hat{a}, 0) w^{*}, w^{*}\right)}{1+2 i<(\overline{\hat{a}}, 0), z^{*}>-i|\hat{a}|^{2} w^{*}}
$$

where $\hat{a}$ is chosen so that the first $\kappa_{0}$ components of the vector:

$$
\frac{\partial^{2} f_{p}^{* * *}}{\partial w^{2}}(0)=i \hat{a} U_{(p, k)} \mathcal{A}(p) U_{(p, k)}^{-1}+\frac{\partial^{2} f_{p}^{* *}}{\partial w^{2}}(0) U_{(p, k)}^{-1}
$$

are zero. (See Lemma 2.2(B), especially (2.5.4)). Write $\eta=\hat{a} U_{(p, k)}$. We can uniquely choose $\hat{a}$ so that $\eta=\left(\eta_{1}, \cdots, \eta_{\kappa_{0}}, 0, \cdots, 0\right) . \eta$ is then also uniquely determined.

By the way $\hat{a}$ is chosen, we have $\frac{\partial^{2} f_{p}^{* * *}}{\partial w^{2}}(0)=0$. It thus follows from $(3.6 .0)^{\prime}$ that

$$
\eta \cdot \mathcal{A}(p)=i \frac{\partial^{2} f_{p}^{* *}}{\partial w^{2}}(0)
$$


Hence, as in (3.4.4), we conclude that $\eta_{j}=\frac{i}{\mu_{j}(0)} T^{2}\left(f_{p, j}^{* *}\right)(0)+T^{2}\left(f_{p}^{* *}\right)(0) \cdot o(1)$, and depends holomorphically on $\left\{T^{2}\left(f_{p}^{* *}\right)(0), P_{j}^{l}(F)\right\}$. Moreover by (3.1.2) and (2.1.5) (2.1.6), we have

$$
\begin{aligned}
& T^{2}\left(f_{p, j}^{* *}\right)(0)=\frac{1}{\lambda(p)}\left(T^{2}(\tilde{f}) \cdot \overline{L_{j} \tilde{f}}-\frac{1}{\lambda}(T \widetilde{f}) \cdot \overline{L_{j} \widetilde{f}}\left(T^{2}(g)-2 i T^{2}(\tilde{f}) \cdot \overrightarrow{\widetilde{f}}\right)\right) \\
& \eta_{j}=\frac{i}{\lambda(p) \mu_{j}}\left(T^{2}(\widetilde{f}) \cdot \overline{L_{j} \tilde{f}}-\frac{1}{\lambda}(T \widetilde{f}) \cdot \overline{L_{j} \widetilde{f}}\left(T^{2}(g)-2 i T^{2}(\tilde{f}) \cdot \overline{\widetilde{f}}^{t}\right)\right)+ \\
& +T^{2}\left(f_{p}^{* *}(0)\right) \cdot o(1) \\
& \overline{L_{k}}\left(\eta_{j}\right)(0)=\frac{i}{\mu_{j}} T^{2}\left(\phi_{j k}\right)(0) \overline{\mu_{j k}}
\end{aligned}
$$

Next notice that $\left(\phi_{p}^{* * *}\right)_{z_{k} w}^{\prime \prime}(0)=\frac{1}{\left|I_{k}(p)\right|}\left(\sum_{j} \xi_{j}\left(\phi_{p}^{* *}\right)_{z_{j} w}^{\prime \prime}(0)+\sum_{j, l} \xi_{j} \eta_{l}\left(\phi_{p}^{* *}\right)_{z_{j}}^{\prime \prime} z_{l}(0)\right) \times$ $\times{\widetilde{U_{p}}}^{-1}=0$; for, by Lemma $2.2(\mathrm{~B}), F_{p}^{* * *}$ satisfies the normalization in (3.2). Arguing the same way as in the proof of Lemma 3.1, by (2.3.4) and formulas in (2.1.2)-(2.1.6), we have the following:

$$
\left(\sum_{j^{\prime}, l=1}^{n-1} \xi_{j^{\prime}} \eta_{l} L_{l} L_{j^{\prime}}(\tilde{f})+L_{\xi} T(\tilde{f})-\frac{2 i}{\lambda(p)} \overline{T(\widetilde{f})} \cdot L_{\xi}(\tilde{f})^{t} T(\tilde{f})\right) \cdot \overline{C_{j}(p)^{t}}=0
$$

for any $j$. Here, we write $L_{\xi}=\sum \xi_{j} L_{j}$. Thus there are $\Omega_{j}(p)^{\prime} s$ such that

$$
\begin{aligned}
& \sum_{j, l} \xi_{j} \eta_{l} L_{j} L_{l}(\tilde{f})+L_{\xi} T(\tilde{f})-\frac{2 i}{\lambda(p)} \overline{T(\widetilde{f})} \cdot L_{\xi}(\tilde{f})^{t} T(\tilde{f})= \\
& =\sum_{j=1}^{n-1} \Omega_{j}(p) E_{j}(p)=\sum_{j=1}^{n-1} \Omega_{j}(p) L_{j}(\widetilde{f})(p) \\
& \text { with } \Omega_{j}(p)= \\
& \left.=\frac{1}{\lambda(p)}\left(\sum_{j, l} \xi_{j^{\prime}} \eta_{l} L_{j^{\prime}} L_{l}(\tilde{f})\right)+L_{\xi} T(\tilde{f})-\frac{2 i}{\lambda(p)} \overline{T(\widetilde{f})} \cdot L_{\xi}(\widetilde{f})^{t} T(\widetilde{f})\right) \cdot \overline{L_{j}(\widetilde{f})^{t}}
\end{aligned}
$$

Making use of the fact that $\sum_{l=1}^{n-1} \xi_{l} P_{l}^{j}(p)=0$ and (3.4.4), we notice that $\Omega_{j}$ is also given by the following:

$$
\Omega_{j}(p)=\frac{\xi_{j}}{2 \lambda(p)}\left(T^{2}(g)-2 i T^{2}(\tilde{f}) \cdot \overline{\widetilde{f}}\right)+\frac{1}{\lambda(p)} \sum_{j^{\prime}, l} \xi_{j^{\prime}} \eta_{l} L_{j^{\prime}} L_{l}(\tilde{f}) \cdot \overline{L_{j}(\widetilde{f})} .
$$

Applying $\overline{L_{k}}$ to (3.6.2) with an application of Claim 3.4, we obtain

$$
\begin{aligned}
& \sum_{j, l=1}^{n-1} \overline{L_{k}}\left(\xi_{j} \eta_{l}\right) L_{j} L_{l}(\tilde{f})+2 i \sum_{l=1}^{n-1} \eta_{l} T L_{l}(\widetilde{f})+2 i \sum_{j, l=1}^{n-1} \xi_{j} \eta_{k} L_{j} T(\tilde{f})+2 i T^{2}(\widetilde{f}) \\
& =\sum_{j=1}^{n-1} \overline{L_{k}}\left(\Omega_{j}\right) L_{j}(\tilde{f})+2 i \Omega_{k} T(\tilde{f})+\overline{L_{k}}\left(\frac{2 i}{\lambda(p)} \overline{T(\widetilde{f})} \cdot L_{\xi}(\tilde{f}) t T(\tilde{f})\right)- \\
& -\sum \overline{L_{k}}\left(\xi_{j}\right) L_{j} T(\tilde{f})
\end{aligned}
$$


Still assume that $F$ is as in Lemma 3.2. Considering the $\phi_{j l}$-component in (3.6.3) and then evaluating at 0 , we have

$$
\sum_{l^{\prime}=1}^{\kappa_{0}} \overline{L_{k}}\left(\eta_{l^{\prime}}\right)(0) L_{k} L_{l^{\prime}}\left(\phi_{j l}\right)(0)+2 i T^{2}\left(\phi_{j l}\right)(0)=0 .
$$

Together with Lemma 3.3(C) and (3.6.1), the above formular gives immediately the following:

$$
T^{2}\left(\phi_{j l}\right)(0)=0 \text { for any }(j, l) \in \mathcal{S} \text {. }
$$

With (3.6.4) and Lemma 3.3 at our disposal, we now are ready to give the following approximate partial linearity theorem for $F$ :

TheOREM 3.5. Suppose that $F$ is a $C^{2}$-smooth $C R$ map from a connected open subset $M$ of 0 in $\mathbf{H}_{n}$ into $\mathbf{H}_{N}$. Suppose that $R k_{F}(p) \equiv \kappa_{0}<n-1$ is constant for any $p \in M$.

$(A):$ Then for any $p \in M$, there is a unique affine complex subspace $S_{p}$ of dimension $\left(n-\kappa_{0}\right)$ transversal to $\mathbf{H}_{n}$ at $p$ such that there is a linear fractional map $\mathcal{L}_{p}$ which maps $S_{p} \cap \mathbf{H}_{n}$ into $\mathbf{H}_{N}$ with the following property

$$
F(Z)-\mathcal{L}_{p}(Z)=o\left(|Z-p|^{2}\right) \text { for } Z \in S_{p} \cap \mathbf{H}_{n} .
$$

(B): For any affine complex line $C_{p}$ transversal to $\mathbf{H}_{n}$ at $p$, if there is a linear fractional map $\Psi_{p}$ sending $C_{p} \cap \mathbf{H}_{n}$ into $\mathbf{H}_{N}$ such that $F(Z)-\Psi_{p}(Z)=o\left(|Z-p|^{2}\right)$ for $Z \in$ $C_{p} \cap \mathbf{H}_{n}$, then $C_{p} \subset S_{p}$.

Proof of Theorem 3.5. We can assume that $F$ satisfies the normalization condition (3.2). Also, from (2.2.3) and (3.6.0), there are $\widetilde{\sigma_{p}^{0}} \in \operatorname{Aut}\left(\mathbf{H}_{n}\right)$ and $\widetilde{\tau_{p}^{F}} \in \operatorname{Aut}\left(\mathbf{H}_{N}\right)$ such that

$$
F_{p}^{* * *}=\widetilde{\tau_{p}^{F}} \circ F \circ \widetilde{\sigma_{p}^{0}} \text { with } \widetilde{\sigma_{p}^{0}}(0)=p, \widetilde{\tau_{p}^{F}}(F(p))=0 \text {. More precisely }
$$

$$
\widetilde{\sigma_{p}^{0}}=\sigma_{p}^{0} \circ \mathcal{U}_{(p, \kappa)} \circ \hat{\sigma_{p}}
$$

where $\sigma_{p}^{0}$ is as in (2.1.0), $\hat{\sigma_{p}}$ is as in (3.6.0) and

$$
\mathcal{U}_{(p, \kappa)}(z, w)=(z, w)\left(\begin{array}{cc}
U_{(p, k)} & 0 \\
0 & 1
\end{array}\right) \text { with } I_{k}(p)=\left|I_{k}(p)\right| e_{k}^{\prime} U_{(p, k)}
$$

Notice that $F_{p}^{* * *}$ satisfies the normalization condition in (3.2). Let $S_{0}=\{Z=$ $\left.\left(z_{1}, \cdots, z_{n-1}, w\right): z_{1}=\cdots=z_{\kappa_{0}}=0\right\}$ be as before and let

$$
\mathcal{L}_{0}\left(z_{1}, \cdots, z_{n-1}, w\right)=\left(0, \cdots, 0, z_{\kappa_{0}+1}, \cdots, z_{n-1}, 0, \cdots, 0, w\right) .
$$

By Lemma 3.3 and (3.6.4), if we define $S_{p}=\widetilde{\sigma_{p}^{0}}\left(S_{0}\right)$ and $\mathcal{L}_{p}=\left(\widetilde{\tau_{p}^{F}}\right)^{-1} \circ \mathcal{L}_{0} \circ{\widetilde{\left(\sigma_{p}^{0}\right)}}^{-1}$, then (3.7.1) holds. To prove the uniqueness of $S_{p}$, it suffices for us to prove the statement in (B) with $p=0$. This can be trivially seen as follows: 
Suppose that $C_{0}$ is parametrized by $z=\left(a_{1}, \cdots, a_{n-1}\right) t=a t, w=t$ and suppose $\left.\Psi_{0}\right|_{C_{0}}=\frac{b t}{1+b_{0} t}=b t-b_{0} b t^{2}+o\left(t^{2}\right)$. Since, when restricted to $C_{0}$,

$$
F=(a t, 0, t)+\frac{i}{2}\left(a_{1} \mu_{1}, \cdots, a_{\kappa_{0}} \mu_{\kappa_{0}}, 0, \cdots, 0, \cdots, a_{j} a_{l}+*, \cdots, 0\right) t^{2}+o\left(t^{2}\right)
$$

it follows that

$$
b=\left(a, 0^{\prime}, 1\right), \quad \frac{i}{2}\left(a_{1} \mu_{1}, \cdots, a_{\kappa_{0}} \mu_{\kappa_{0}}, 0, \cdots, a_{j} a_{l}+*, 0 \cdots, 0\right)=-b_{0} b .
$$

Thus, we conclude that $b_{0}=0, a_{j}=0$ for $j \leq \kappa_{0}$. Namely, $C_{0} \subset S_{0}$. Finally, we emphasize again the fact that

$$
S_{p}=\widetilde{\sigma_{p}^{0}}\left(S_{0}\right)
$$

which will be used later. The proof of Theorem 3.5 is complete.

For each $p \in M$, let $S_{p}$ be as in Theorem 3.5. Define $\mathcal{V}_{F}(p)=C T_{p}\left(S_{p} \cap \mathbf{H}_{n}\right)$ and write $\mathcal{V}_{F}$ for the vector subbundle of $C T M$, whose fiber at $p$ is $\mathcal{V}_{F}(p)$. In what follows, for an $(n-1)$-vector valued function $J(p)=\left(v_{1}(p), \cdots, v_{n-1}(p)\right)$ along $M$, we write $J^{L}$ for the vector field $\sum_{j=1}^{n-1} v_{j}(p) L_{j}$. We call that $\mathcal{V}_{F}$ is integrable in the strong sense if $M$ can be foliated by $\mathcal{V}_{F}$-integrable $C^{2}$ submanifolds of real dimension $2\left(n-\kappa_{0}\right)-1$. The study of the strong integrability of $\mathcal{V}_{F}$ is closely related to the partial linearity of $F$. We will not address this issue here. Instead, we will be content with presenting the following proposition, which gives a partial integrability statement for the distribution $\Gamma\left(\mathcal{V}_{F}\right)$.

Proposition 3.6. Let $F$ be as in Theorem 3.5. Assume the notation we just set up. Write $\mathcal{V}_{F}^{(1,0)}=\mathcal{V}_{F} \cap T^{(1,0)} M$.

(I). Then the space $\Gamma\left(\mathcal{V}_{F}^{(1,0)}\right)$ of cross sections of $\mathcal{V}^{(1,0)}$ has $\left\{I_{k}^{L}\right\}_{k=\kappa_{0}+1, \cdots, n-1}$ as its basis.

(II). For each $k^{\prime}>\kappa_{0}, \Gamma\left(\mathcal{V}_{F}\right)$ has $\left\{I_{\kappa_{0}+1}^{L}, \overline{I_{\kappa_{0}+1}^{L}}, \cdots, I_{n-1}^{L}, \overline{I_{n-1}^{L}},\left[I_{k^{\prime}}^{L}, \overline{I_{k^{\prime}}^{L}}\right]\right\}$ as its basis. Moreover, for each $k^{\prime}, k^{\prime \prime}>\kappa_{0}$, it holds that

$$
\left.\left[I_{k^{\prime}}^{L}, \overline{I_{k^{\prime \prime}}^{L}}\right],\left[I_{k^{\prime}}^{L}, I_{k^{\prime \prime}}^{L}\right], \overline{\left[I_{k^{\prime}}^{L}\right.}, \overline{I_{k^{\prime \prime}}^{L}}\right] \in \Gamma\left(\mathcal{V}_{F}\right)
$$

(III) Let $S_{p}$ be as in Theorem 3.5. Then $S_{p}-p$, as an element in the complex Grassmannian manifold $G_{n, n-\kappa_{0}}(\mathbf{C})$ of $\left(n-\kappa_{0}\right)$-dimensional linear subspaces in $\mathbf{C}^{n}$, depends continuously on $p$. Moreover, if $F \in C^{k}(M)$, then $S_{p}$ depends $C^{k-2}$-continuously on $p$.

Proof of Proposition 3.6. Let $\widetilde{\sigma_{(p, k)}^{0}}$ be as in (3.7.2). (Here we put the index $k$ to emphasize its dependence on $k$ ). Then

$$
\mathcal{V}_{F}^{(1,0)}(p)=\operatorname{span}\left\{D_{0}\left(\widetilde{\sigma_{(p, k)}^{0}}\right)\left(\left.e_{k}^{\prime L}\right|_{0}\right)\right\}_{k=\kappa_{0}+1, \cdots, n-1},
$$

where $D_{0}(\cdot)$ denotes the differential push-forward map. Notice that $\hat{\sigma}_{p}(0)=0$, $\left.D_{0}\left(\hat{\sigma}_{p}\right)\right|_{T_{0}^{(1,0)}(M)}=\mathrm{Id}, \sigma_{p}^{0}$ is as defined in (2.1.0). 
In what follows, we start to write $I_{k}=\left(\xi_{j}^{k}\right)_{1 \leq k \leq n-1}$ to emphasize its dependence on the index $k$. Then

$$
\begin{aligned}
& D_{0}\left(\widetilde{\sigma_{(p, k)}^{0}}\right)\left(\left.e_{k}^{\prime L}\right|_{0}\right)=D_{0}\left(\sigma_{p}^{0} \circ \mathcal{U}_{(p, k)}^{0}\right)\left(\left.e_{k}^{{ }^{L}}\right|_{0}\right) \\
& =\frac{1}{\left|I_{k}(p)\right|} D_{0}\left(\sigma_{p}^{0}\right)\left(\left.\sum \xi_{j}^{k}(p) e_{j}^{\prime L}\right|_{0}\right)=\left.\frac{1}{\left|I_{k}(p)\right|} \sum \xi_{j}^{k}(p) e_{j}^{\prime L}\right|_{p}=\frac{I_{k}^{L}(p)}{\left|I_{k}(p)\right|} .
\end{aligned}
$$

In terms of (3.7.4), this gives the proof of the statement in (I).

Next, since $P_{j}^{l}=-\overline{P_{l}^{j}}$, by (3.4.3) and (3.4.4), for $Z_{0} \in S_{0}$, we have

$$
\xi_{j}^{k}\left(Z_{0}\right)=\frac{-2 i}{\mu_{j}} \overline{P_{j}^{k}\left(Z_{0}\right)}+o\left(\left|\sum_{l=1}^{\kappa_{0}} P_{l}^{k}\left(Z_{0}\right)\right|\right)=\frac{-2 i}{\mu_{j}} \overline{L_{j} T\left(f_{k}\right)\left(Z_{0}\right)}+o_{w t}(1)
$$

We conclude that

$$
L_{k^{\prime}}\left(\xi_{j}^{k}\right)(0)=0
$$

Notice that $\overline{L_{k^{\prime}}}\left(\xi_{j}^{k}\right)(0)=0$ by (3.5.3). We can easily arrive at the following:

$$
\mathcal{V}_{F}(0)=\operatorname{span}\left\{\left.I_{\kappa_{0}+1}^{L}\right|_{0},\left.\overline{I_{\kappa_{0}+1}^{L}}\right|_{0}, \cdots,\left.I_{n-1}^{L}\right|_{0},\left.\overline{I_{n-1}^{L}}\right|_{0},\left.\left[I_{k^{\prime}}^{L}, \overline{I_{k^{\prime}}^{L}}\right]\right|_{0}\right\}
$$

Moreover, for each $k^{\prime}, k^{\prime \prime}>\kappa_{0}$, it holds that

$$
\left.\left[I_{k^{\prime}}^{L}, \overline{I_{k^{\prime \prime}}^{L}}\right]\right|_{0},\left.\left[I_{k^{\prime}}^{L}, I_{k^{\prime \prime}}^{L}\right]\right|_{0},\left.\left[\overline{I_{k^{\prime}}^{L}}, \overline{I_{k^{\prime \prime}}^{L}}\right]\right|_{0} \in \mathcal{V}_{F}(0)
$$

Notice that $\left\{\left.I_{\kappa_{0}+1}^{L}\right|_{0},\left.\overline{I_{\kappa_{0}+1}^{L}}\right|_{0}, \cdots, I_{n-1}^{L}\left|0, \overline{I_{n-1}^{L}}\right|_{0},\left.\left[I_{k^{\prime}}^{L}, \overline{I_{k^{\prime}}^{L}}\right]\right|_{0}\right\}$ must be a basis for $\left.\mathcal{V}_{F}\right|_{0}$; for it is a linearly independent system and has the right dimension. This proves the statement in (II) at the origin.

To achieve (3.8.3) (3.8.4) at any other point $p_{0}(\approx 0) \in M$, we need to go back to the map $F_{p_{0}}^{* * *}$ defined in (3.7.2). Write $p^{\prime}=\widetilde{\sigma_{\left(p_{0}, k\right)}^{0}}{ }^{-1}(p)$ or $p=\widetilde{\sigma_{\left(p_{0}, k\right)}^{0}}\left(p^{\prime}\right)$. For $p \approx p_{0}$, notice that $\widetilde{J}_{k}^{L}:=D_{p^{\prime}}\left(\widetilde{\sigma_{\left(p_{0}, k\right)}^{0}}\right)\left(e_{k}^{\prime L}\left(p^{\prime}\right)\right)$ is a smooth $(1,0)$-type vector field, which, when restricted to $S_{p_{0}} \cap \mathbf{H}_{n}$, must be tangent to $S_{p_{0}} \cap \mathbf{H}_{n}$ near $p_{0}$, by the way $S_{p_{0}}$ was constructed. Write $S_{F_{p_{0} * *}^{* *}}\left(p^{\prime}\right)$ for the affine complex subspace of dimension $\left(n-\kappa_{0}\right)$ along which $F_{p_{0}}^{* * *}$ is approximated by a linear fractional map as in Theorem 3.5. (Apparently, we can apply Theorem 3.5 to $F_{p_{0}}^{* * *}$.) Then it is clear that $\widetilde{\sigma_{\left(p_{0}, k\right)}^{0}}\left(S_{F_{p_{0}}^{* * *}}\left(p^{\prime}\right)\right)=S_{F}(p)$ and thus $D_{p^{\prime}}\left(\widetilde{\sigma_{\left(p_{0}, k\right)}^{0}}\right)\left(\mathcal{V}_{F_{p_{0}}^{* * *}}^{(1,0)}\left(p^{\prime}\right)\right)=\mathcal{V}_{F}^{(1,0)}(p)$. Hence, there are functions $b_{k, k^{\prime}}(p)^{\prime} s$ such that

$$
I_{k}^{L}(p)=\sum_{k^{\prime}=\kappa_{0}+1}^{n-1} b_{k, k^{\prime}}(p) D_{p^{\prime}}\left(\widetilde{\sigma_{\left(p_{0}, k\right)}^{0}}\right)\left(I_{k^{\prime}}^{L}\left(p^{\prime}, F_{p_{0}}^{* * *}\right)\right),
$$

where $I_{k^{\prime}}^{L}\left(p^{\prime}, F_{p_{0}}^{* * *}\right)$ is the corresponding $I_{k^{\prime}}^{L}$-vector field associated to the map $F_{p_{0}}^{* * *}$ at $p^{\prime}$. Also, $b_{k, k^{\prime}}(p)$ can be uniquely solved by noting the fact that $\left\{I_{k}^{L}\left(p^{\prime}, F_{p_{0}}^{* * *}\right)\right\}_{k=\kappa_{0}+1}^{n-1}$ is a linearly independent system for $p^{\prime} \approx 0$. In fact, it can be seen that

$$
b_{k, k^{\prime}}(p)=B_{k, k^{\prime}}\left(I_{k^{\prime \prime}}(p), I_{k^{\prime \prime}}\left(p^{\prime}, F_{p_{0}}^{* * *}\right), p, \bar{p}\right)
$$


for a certain $B_{k, k^{\prime}}$ holomorphic in its own variables. By Lemma 3.3 (A) and (3.8.6), $L_{j}\left(b_{k, k^{\prime}}(p)\right)$ and $\overline{L_{j}}\left(b_{k, k^{\prime}}(p)\right)$ are continuous near $p_{0}$. Now, write

$$
J_{k}^{L}(p)=\sum_{k^{\prime} \geq \kappa_{0}+1} b_{k, k^{\prime}}(p) \widetilde{J_{k^{\prime}}^{L}}(p)
$$

Then $J_{k}^{L}$ is tangent to $S_{p_{0}} \cap \mathbf{H}_{n}$ near $p_{0}$ and

$$
\begin{aligned}
I_{k}^{L}-J_{k}^{L} & =\sum_{k^{\prime}=\kappa_{0}+1}^{n-1} b_{k, k^{\prime}}(p) D_{p^{\prime}}\left(\widetilde{\sigma_{\left(p_{0}, k\right)}^{0}}\right)\left(I_{k^{\prime}}^{L}\left(p^{\prime}, F_{p_{0}}^{* * *}\right)-e_{k}^{\prime L}\left(p^{\prime}\right)\right) \\
& =\sum_{k^{\prime}=\kappa_{0}+1}^{n-1} b_{k, k^{\prime}}(p) D_{p^{\prime}}\left(\widetilde{\sigma_{\left(p_{0}, k\right)}^{0}}\right)\left(\xi_{j}^{k}\left(p^{\prime}, F_{p_{0}}^{* * *}\right) L_{j}\left(p^{\prime}\right)\right) .
\end{aligned}
$$

Write $D_{p^{\prime}}\left(\widetilde{\sigma_{\left(p_{0}, k\right)}^{0}}\right)\left(L_{j}\left(p^{\prime}\right)\right)=\sum_{j^{\prime}} c_{j^{\prime}, j} L_{j^{\prime}}(p)$. Then

$$
I_{k}^{L}-J_{k}^{L}=\sum_{j} \chi_{j}^{k}(p) L_{j}
$$

with

$$
\left.\chi_{j}^{k}(p)=\sum_{k^{\prime}=\kappa_{0}+1, \cdots, n-1 ; j^{\prime}=1, \cdots, n-1} b_{k, k^{\prime}} \xi_{j^{\prime}}^{k^{\prime}}\left(\widetilde{\sigma_{\left(p_{0}, k\right)}^{0}}\right)^{-1}(p), F_{p_{0}}^{* * *}\right) c_{j, j^{\prime}}(p)
$$

Hence, $L_{l}\left(\chi_{j}^{k}(p)\right)$ and $\overline{L_{l}}\left(\chi_{j}^{k}(p)\right)$ continuous near $p_{0}$. Moreover, we have

$$
\chi_{j}^{k}\left(p_{0}\right)=J_{k^{\prime}}^{L}\left(\chi_{j}^{k}\right)\left(p_{0}\right)=\overline{J_{k^{\prime}}^{L}}\left(\chi_{j}^{k}\right)\left(p_{0}\right)=0,
$$

by $(3.8 .2)^{\prime}$ and $(3.5 .3)$ (but for $\left.I_{k}\left(\cdot, F_{p_{0}}^{* * *}\right)\right)$. Hence,

$$
\begin{aligned}
{\left[I_{k^{\prime}}^{L}, \overline{I_{k^{\prime \prime}}^{L}}\right]\left(p_{0}\right) } & =\left.\left(\left[J_{k^{\prime}}^{L}, \overline{J_{k^{\prime \prime}}^{L}}\right]+\sum_{j, l^{\prime}} \overline{J_{k^{\prime \prime}}^{L}}\left(\chi_{l}^{k^{\prime}}\right) L_{l}-\sum_{j, l} J_{k^{\prime \prime}}^{L} \overline{\left(\chi_{l}^{k^{\prime}}\right)} \overline{L_{l}}+o_{w t}(0)\right)\right|_{p_{0}} \\
& =\left[J_{k^{\prime}}^{L}, \overline{J_{k^{\prime \prime}}^{L}}\right]\left(p_{0}\right)
\end{aligned}
$$

by making use of (3.8.9). Notice that $\left[J_{k^{\prime}}^{L}, \overline{J_{k^{\prime \prime}}^{L}}\right]\left(p_{0}\right) \in \mathcal{V}_{F}\left(p_{0}\right)$ and $\left[J_{k^{\prime}}^{L}, \overline{J_{k^{\prime}}^{L}}\right]\left(p_{0}\right) \notin$ $\mathcal{V}_{F}^{(1,0)}\left(p_{0}\right) \cup \mathcal{V}_{F}^{(0,1)}\left(p_{0}\right)$, we conclude the first statement in (II) by counting the dimension.

Similarly, we can verify the other identities in Proposition 3.6 (II).

By the results in (I) and (II), we notice that the complex linear space $T_{p}^{(1,0)}\left(S_{p}\right)$ has basis

$$
\left\{I_{\kappa_{0}+1}^{L}(p), \cdots, I_{n-1}^{L}(p),\left[I_{n-1}^{L}, \overline{I_{n-1}^{L}}\right](p)-i \mathcal{J}\left(\left[I_{n-1}^{L}, \overline{I_{n-1}^{L}}\right]\right)(p)\right\}
$$

where $\mathcal{J}$ is the standard complex structure in $\mathbf{C}^{n}$. We easily conclude the statement in (III).

The proof of Proposition 3.6 is complete. 
4. Proof of Theorem 2.3. In this section, with the preparation in $\S 3$, we will complete the proof of Theorem 2.3. Since $F$ is now assumed to be $C^{3}$, there are two ways to achieve it. One way is to continue the study of the integrability of $\mathcal{V}_{F}$ and then restrict the equation (3.1.0) to each leaf to reduce the linearity problem to the uniqueness of differential equations. Another method is to obtain the linearity by reducing the problem to a boundary uniqueness problem for holomorphic functions, as in $\S 4$ of [HJ]. Here, we present a discussion on the latter approach, which mainly depends on results obtained in $\S 3.1$.

We now proceed to explain how the proof of Theorem 2.3 can be obtained by reducing the problem to the uniqueness problem of holomorphic functions so that we can apply a version of the classical Hopf Lemma due to Burns-Krantz. This approach was motivated by the argument in $\S 4$ of [HJ]. We first start with the following lemma, for whose proof one just needs to copy the argument from [Lemma 5.3, Hu1] with a notice of the $C^{l}$-smoothness assumption for the map here.

LemMA 4.0. Let $F$ be a $C^{l}$-smooth $C R$ map from a neighborhood $M$ of $0 \in \mathbf{H}_{n}$ into $\mathbf{H}_{N}$ with $l \geq 2$. Assume that $F$ satisfies the normalization condition (2.2.1). Then

$$
g \in \mathcal{P}+o_{w t}(l+2), f \in \mathcal{P}+o_{w t}(l+1)
$$

For the rest of this section, we will assume that $F$ is $C^{3}$-smooth.

We next present the following generalization of Lemma 3.2 of [HJ].

Lemma 4.1. Let $F=(f, \phi, g)$ be a $C^{3}$-smooth CR map from $M$ into $\mathbf{H}_{N}$, which satisfies the normalization condition (3.2). Then $f \in \mathcal{P}+o_{w t}(4), g \in \mathcal{P}+o_{w t}(5)$. Moreover, we have the following weighted decomposition:

$f_{j}^{(4)}=a_{j}^{(2)}(z) w+a_{02, j} w^{2}, g^{(5)}=c^{(1)}(z) w^{2}, \phi_{j l}=\phi_{j l}^{(2)}(z)+b_{j l}^{(3)}(z)+b_{j l}^{(1)}(z) w+o_{w t}(3)$

with

$$
\begin{aligned}
& a_{02, j}=0 \text { for } j \leq n-1, c^{(1)}(z) \equiv 0, \frac{\partial b_{j l}^{(1)}(z)}{\partial z_{k}}=0 \text { for } k>\kappa_{0} \text { and }(j, l) \in \mathcal{S}_{0} \\
& a_{j}^{(2)}(z)=0 \text { for } j \geq \kappa_{0}+1, \text { and } a_{j}\left(0, \cdots, 0, z_{\kappa_{0}+1}, \cdots, z_{n-1}\right)=0 \text { for } j \leq \kappa_{0} .
\end{aligned}
$$

Proof of Lemma 4.1. Applying Lemma 4.0, we can conclude that $f \in \mathcal{P}+o_{w t}(4)$ and $g \in \mathcal{P}+o_{w t}(5)$.

Collecting terms of weighted degree 5 in the basic equation $\operatorname{Im}(g)=|\widetilde{f}|^{2}$ for $\operatorname{Im}(w)=|z|^{2}$, we obtain the equation:

$$
\operatorname{Im}\left(g^{(5)}-2 i \bar{z} \cdot f^{(4)}\right)=2 \operatorname{Re}\left(\phi^{2} \cdot \overline{\phi^{(3)}}\right) .
$$

As in [Lemma 3.2, HJ], it is easy to conclude from (4.3) the expansion in (4.1). Moreover, letting $z_{1}=\cdots=z_{\kappa_{0}}=0$ in (4.3) and applying the uniqueness of the ChernMoser Lemma ([Lemma 3.1, CH] (or by a direct calculation), we have that $a_{02, j}=0$ 
for $j \geq \kappa_{0}+1$. Collecting coefficients of $u^{2}$ in $(4.3)$, we get $c^{(1)}(z)=2 i \sum_{j=1}^{\kappa_{0}} \overline{a_{02, j}} z_{j}$. Since $a_{02, j}=0$ for $j \leq \kappa_{0}$ by the normalization in (3.2), we see that $g^{(5)}=0$. Hence, (4.3) can be simplified as follows:

$$
\operatorname{Im}\left(2 i \sum_{j=1}^{n-1} \overline{z_{j}} \cdot a_{j}^{(2)}(z) w+2 i \sum_{(j, l) \in \mathcal{S}_{0}} \phi_{j l}^{(2)} \cdot \overline{\left(b_{j l}^{(3)}(z)+w b_{j l}^{(1)}(z)\right)}\right)=0, \quad w=u+i|z|^{2} .
$$

Collecting the coefficients of $u$ in (4.4), we get $\sum_{j=1}^{n-1} \overline{z_{j}} \cdot a_{j}^{(2)}(z)=-\sum_{(j, l) \in \mathcal{S}_{0}} \phi_{j l}^{(2)}(z)$. $\overline{b_{j l}^{(1)}(z)}$. Thus, we get

$$
a_{m}^{(2)}=-\sum_{(j, l) \in \mathcal{S}_{0}} \phi_{j l}^{(2)}(z) \overline{\frac{\partial b_{j l}^{(1)}(z)}{\partial z_{m}}} .
$$

By the normalization in $(3.2)$, we get $a_{m}^{(2)}\left(0, \cdots, 0, z_{\kappa_{0}+1}, \cdots, z_{n-1}\right)=0$ for any $m$. Collecting terms in (4.4) without the $u$-factor, we get

$$
\sum_{j=1}^{n-1} \overline{z_{j}} a_{j}^{(2)}(z)|z|^{2}=\sum_{(j, l) \in \mathcal{S}_{0}} \phi_{j l}^{(2)}(z) \overline{b_{j l}^{(1)}(z)}|z|^{2}+i \sum_{(j, l) \in \mathcal{S}_{0}} \overline{\phi_{j l}^{(2)}(z)} b_{j l}^{(3)}(z) .
$$

Collecting terms in the above equation with the factor $\left|z_{k}\right|^{2} \overline{z_{k}}$ with $k>\kappa_{0}$, we have

$$
a_{k}^{(2)}(z)=\sum_{(j, l) \in \mathcal{S}_{0}} \phi_{j l}^{(2)}(z) \overline{\frac{\partial b_{j l}^{(1)}(z)}{\partial z_{k}}}
$$

Combining (4.5) with (4.7), we thus obtain $a_{k}^{(2)}(z)=0$ and $\frac{\partial b_{j l}^{(1)}}{\partial z_{k}}=0$ for $(j, l) \in \mathcal{S}_{0}$ and $k>\kappa_{0}$.

We now let $F$ be as in Theorem 2.3 with constant geometric rank $\kappa_{0}$ over $M$. Without loss of generality, we further assume that $0 \in M, n-1>\kappa_{0}>0$ and $F$ satisfies the normalization in (3.2). Also, we keep the notations which we have set up. Write

$$
\mathbf{q}=\left(0, \cdots, 0, q_{1}, \cdots, q_{n-\kappa_{0}-1}\right) \in \mathbf{C}^{n-1} .
$$

Let $F_{c}^{q}=\tau_{c}^{q} \circ F \circ \sigma_{c}^{q}$, where the two automorphisms $\sigma_{c}^{q} \in \operatorname{Aut}\left(\mathbf{H}_{n}\right)$ and $\tau_{c}^{q} \in \operatorname{Aut}\left(\mathbf{H}_{N}\right)$ are given by

$$
\sigma_{c}^{q}(z, w)=\left(\frac{z+w \mathbf{q}}{1-2 i \overline{\mathbf{q}} z-i|\mathbf{q}|^{2} w}, \frac{w}{1-2 i \overline{\mathbf{q}} z-i|\mathbf{q}|^{2} w}\right)
$$

$$
\tau_{c}^{q}\left(z^{*}, w^{*}\right)=\left(\frac{z^{*}-w^{*}\left(\mathbf{q}, 0^{\prime}\right)}{1+2 i<\left(\overline{\mathbf{q}}, 0^{\prime}\right), z^{*}>-i|\mathbf{q}|^{2} w^{*}}, \frac{w^{*}}{1+2 i<\left(\overline{\mathbf{q}}, 0^{\prime}\right), z^{*}>-i|\mathbf{q}|^{2} w^{*}}\right) .
$$

Corollary 4.2. Let $F$ be as in (3.2) and let $\sigma_{c}^{q}, \tau_{c}^{q}$ be as in (4.8)-(4.9). Define $F_{c}^{q}:=\tau_{c}^{q} \circ F \circ \sigma_{c}^{q}$, which we write as $F_{c}^{q}=\left(f_{c}^{q}, \phi_{c}^{q}, g_{c}^{q}\right)=\left(\widetilde{f}_{c}^{q}, g_{c}^{q}\right)$ 
$=\left(f_{c, 1}^{q}, \cdots, \phi_{c, j l}^{q}, \cdots, g_{c}^{q}\right)$. Then $F_{c}^{q}$ still satisfies the normalization condition (3.2). Moreover, we have $g_{c}^{q}=w+o_{w t}(5), \frac{\partial^{2} f_{c}^{q}}{\partial w^{2}}(0)=0, \frac{\partial^{2} \phi_{c}^{q}}{\partial w \partial z_{k}}(0)=0$ for $k>\kappa_{0},\left(f_{c}^{q}\right)^{(4)}=$ $\left(a_{c}^{q}\right)^{(2)}(z) w^{2}$ with $\left(a_{c, j}^{q}\right)^{(2)}\left(0, \cdots, 0, z_{\kappa_{0}+1}, \cdots, z_{n-1}\right)=0$ for $j \leq \kappa_{0}$, and $\left(a_{c, j}^{q}\right)^{(2)}=0$ for $j \geq \kappa_{0}+1$.

Proof of Corollary 4.2. By Lemma 2.2 (A) (C), Lemma 3.3 (C), (2.5.4) and a direct verification, we conclude that $F_{c}$ satisfies the normalization condition (3.2). Hence, Corollary 4.2 follows from Lemma 4.1.

Let $S_{0}=\left\{\left(z_{1}, \cdots, z_{n-1}, w\right):\left(z_{1}, \cdots, z_{\kappa_{0}}\right)=0\right\}$ and

$$
S_{0}^{\prime}=\left\{\left(z_{1}^{*}, \cdots, z_{N-1}^{*}, w^{*}\right): z_{1}^{*}=\cdots=z_{\kappa_{0}}^{*}=z_{n}^{*}=\cdots=z_{N-1}^{*}=0\right\} .
$$

Then $S_{0}$ and $S_{0}^{\prime}$ are complex subspaces of dimension $n-\kappa_{0}$ in $\mathbf{C}^{n}$ and $\mathbf{C}^{N}$, respectively. They are also transversal to the corresponding Heisenberg hypersurfaces. With the above preparation, we are ready to prove the following:

LEMmA 4.3. Let $F$ be a $C^{3}$-smooth CR map from a neighborhood of $0 \in \mathbf{H}_{n}$ into $\mathbf{H}_{N}$, that has constant geometric rank $\kappa_{0}>1$ and satisfies the normalization condition (3.2). Assume the above notation. Then $F\left(S_{0} \cap M\right) \subset S_{0}^{\prime} \cap \mathbf{H}_{N}$.

Proof of Lemma 4.3. For $q=\left(q_{1}, \cdots, q_{n-\kappa_{0}-1}\right) \in \mathbf{C}^{n-\kappa_{0}-1}$ with $|q|>>1$, we let $F_{c}^{q}$ be defined as in Corollary 4.2 .

ClaIm 4.4. $g_{c}^{q}(0, w)=w+o\left(w^{3}\right)$.

Proof of Claim 4.4. For a fixed $k>\kappa_{0}$, let $I_{c, k}^{q}=\left\{\left(\xi_{c, j}^{q}\right)\right\}$ be the corresponding vector associated to the map $F_{c}^{q}$. Notice that $F_{c}^{q}$ satisfies the normalization condition in (3.2). Hence, by Lemmas 3.3, 4.1 and Corollary 4.2, it follows that $g_{c}^{q}=w+o_{w t}(5)$, $\xi_{c, j}^{q}=O_{w t}(2), \overline{L_{k}}\left(\xi_{c, j}^{q}\right)=o_{w t}(0)$ for $j \leq \kappa_{0}$. We thus conclude from (3.4.2) the following:

$$
-8 T^{2}\left(g_{c}^{q}\right)=\sum_{l=1}^{n-1} \overline{L_{k}^{2}}\left(\Lambda_{c, l}^{q}\right) 2 i \overline{z_{l}}+4 i \overline{L_{k}}\left(\Lambda_{c, l}^{q}\right)+o_{w t}(2),
$$

where $\Lambda_{c, l}^{q}=\sum_{j=1}^{n-1} \frac{4 \sqrt{-1}}{\lambda_{c}^{q}} \xi_{c, j}^{q} \xi_{c, l}^{q} \overline{\widetilde{\left(f_{c}^{q}\right)_{w}^{\prime}}} \cdot L_{j}\left(\widetilde{f_{c}^{q}}\right)$. In the remaining argument for this claim, we drop the subscript $c$ and superscript $q$ to simplify the notation. Also, for two functions $A(Z)$ and $B(Z)$ with $Z=(z, \bar{z}, u)$, we say $A=B \bmod$ (terms other than $\left.Z^{\alpha_{1}} \overline{Z^{\beta_{1}}}, \cdots, Z^{\alpha_{l}} \overline{Z^{\beta_{l}}}\right)$ if in the weighted expansions of $A(Z)$ and $B(Z)$ up to order $\max _{j}\left\{\operatorname{deg}_{w t}\left(Z^{\alpha_{j}} Z^{\overline{\beta_{j}}}\right)\right\}$, the coefficients for the $Z^{\alpha_{j}} \overline{Z^{\beta_{j}}}$-terms $(j=1, \cdots, l)$ are the same.

Recall by (3.5.1) that $\overline{L_{k}}\left(\Lambda_{l}\right)=I I_{1}+I I_{2}+I I_{3}+I I_{4}$, where

$$
\begin{gathered}
\left.I I_{1}=\sum_{j=1}^{n-1} \frac{-4 i}{\lambda^{2}} \overline{\overline{L_{k}}}(\lambda)\left(\overline{\widetilde{f}_{w}^{\prime}} \cdot L_{j} \tilde{f}^{t}\right) \xi_{j} \xi_{l}, I I_{2}=\sum_{j=1}^{n-1} \frac{4 i}{\lambda} \overline{\left(L_{k}\left(\widetilde{f}_{w}^{\prime}\right)\right.} \cdot L_{j} \tilde{f}^{t}\right) \xi_{j} \xi_{l} \\
\left.I I_{3}=\frac{-8}{\lambda}\left(\overline{\tilde{f}_{w}^{\prime}} \cdot\left(\widetilde{f_{w}^{\prime}}\right)^{t}\right) \xi_{l}, I I_{4}=\sum_{j=1}^{n-1} \frac{4 i}{\lambda} \overline{\left(\widetilde{f_{w}^{\prime}}\right.} \cdot L_{j} \widetilde{f}^{t}\right) \overline{L_{k}}\left(\xi_{j} \xi_{l}\right) .
\end{gathered}
$$


Since we assumed that $F$ is $C^{3}$, it can be easily seen that $\overline{L_{l}}\left(\xi_{j}\right)$ and $L_{l}\left(\xi_{j}\right)$ are $C^{1}$ near the origin. Moreover, by (2.2.1), Lemma 3.3 (A) and Lemma 3.3 (C), we have $\lambda=1+o_{w t}(1), \xi_{j}=O_{w t}(2), \overline{L_{k}}\left(\xi_{j}\right)=O_{w t}(1)$. With these at our dispósal and making use of Lemma 3.3 , one can easily verify that

$$
\begin{aligned}
& I I_{1}, I I_{3}, I I_{4}=o_{w t}(2) \bmod \left(\text { terms other than }\left|z_{k}\right|^{2}, u\right), \\
& I I_{2}=4 i \overline{L_{k}\left(\left(f_{k}\right)_{w}^{\prime}\right)}+o_{w t}(2) \bmod \left(\text { terms other than }\left|z_{k}\right|^{2}, u\right) .
\end{aligned}
$$

Notice that $\overline{L_{j}}\left(L_{k}\left(\left(f_{k}\right)_{w}^{\prime}\right) \in \mathcal{P}+o_{w t}(1), T\left(L_{k}\left(\left(f_{k}\right)_{w}^{\prime}\right)\right) \in \mathcal{P}+o_{w t}(0)\right.$. Also applying $L_{j}$ to the $f_{k}$-component of the first equation in (3.4.1), one sees that $L_{j}\left(L_{k}\left(\left(f_{k}\right)_{w}^{\prime}\right)=\right.$ $L_{j}\left(\overline{L_{k}}\left(\Lambda_{k}\right)\right)+o_{w t}(1) \in \mathcal{P}+o_{w t}(1)$. This shows by [Lemma 5.2, Hu1] that $\left.L_{k}\left(\left(f_{k}\right)_{w}^{\prime}\right)\right) \in$ $\mathcal{P}+o_{w t}(2)$.

Next, write $\gamma=\frac{\partial^{3} f_{k}}{\partial z_{k} \partial w^{2}}(0)$. We thus obtain $I I_{2}=4 i \bar{\gamma}\left(u-3 i\left|z_{k}\right|^{2}\right) \bmod ($ terms other than $\left.\left|z_{k}\right|^{2}, u\right)$. Hence, it follows that

$$
-8 T^{2}(g)=-16\left(\bar{\gamma}\left(u-3 i\left|z_{k}\right|^{2}\right)\right)+32 i \bar{\gamma}\left|z_{k}\right|^{2}+o_{w t}(2) \bmod \left(\text { terms other than }\left|z_{k}\right|^{2}, u\right) \text {. }
$$

Write $c_{03}=\frac{1}{6} \frac{\partial^{3} g}{\partial w^{3}}(0)$. From (4.10), we have $T^{2}(g) \in \mathcal{P}+o_{w t}(2)$. Hence, by a similar argument as above, it follows that

$$
c_{03}\left(u+i\left|z_{k}\right|^{2}\right)=2 \bar{\gamma} u-10 i \bar{\gamma}\left|z_{k}\right|^{2}
$$

which gives immediately that $c_{03}=0$.

Notice that $g_{c}^{q}\left(0^{\prime}, w\right)$ is defined over the upper-half plane for $|q|>>1$. Once we know that $g_{c}^{q}\left(0^{\prime}, w\right)=w+o\left(w^{3}\right)$, by a generalized Hopf lemma due to BurnsKrantz [BK], we can conclude that $g_{c}^{q}\left(0^{\prime}, w\right)=w$ for $|q|>>1$. In fact, for $|q|>>1$, we can define the harmonic function $h$ on the upper-half plane of $\mathbf{C}$ by: $h(w)=$ $\operatorname{Im}\left(\frac{1}{w}-\frac{1}{g_{c}^{q}\left(0^{\prime}, w\right)}\right)$. Then it is clear that $h(w)=o(|w|)$ as $w \rightarrow 0$ and $\lim _{w\left(\in H^{+}\right) \rightarrow x \in(\mathcal{R} \cup \infty)} h(w) \geq 0$. Hence 0 is the minimum value of $h(w)$. By the classical Hopf lemma, it follows that $h(w) \equiv 0$. Namely, $g_{c}^{q}\left(0^{\prime}, w\right) \equiv w$.

Next, we also have $\widetilde{f}_{c}^{q}(0, w) \equiv 0$ by the boundary equation $\operatorname{Im}\left(g_{c}^{q}\right)=\left|\widetilde{f}_{c}^{q}\right|^{2}$. Let $q$ vary. We conclude that $F$ maps an open subset of $S_{0} \cap \mathbf{H}_{n}$ into $S_{0}^{\prime} \cap \mathbf{H}_{N}$. By a theorem of Alexander [Alx], it follows that $F$ must be linear fractional when restricted to $S_{0} \cap$ $\mathbf{S}_{n} \cap \Omega$. Considering $F_{p}^{* * *}$ in (3.6.0) instead of $F$, we conclude that for each $p(\approx 0) \in$ $\mathbf{H}_{n}$, there is a unique affine subspace $S_{p}$ of dimension $n-\kappa_{0}$ such that $\left.F\right|_{S_{p} \cap \Omega}$ is a linear fractional map. (The uniqueness follows from Lemma 2.2(D) or Theorem 3.5). Also, by Proposition 3.6 (III), $S_{p}-p$, as an element in the complex Grassmannian manifold $G_{n, n-\kappa_{0}}(\mathbf{C})$ of $\left(n-\kappa_{0}\right)$-dimensional linear spaces in $\mathbf{C}^{n}$, depends continuously on $p$ (or $C^{l-2}$-smoothly on $p$, if $F$ is assumed to be $C^{l}$ ). (If we just need the dependence in a neighborhood of a certain point $p^{*} \approx p$, we then do not need Proposition 3.6. Indeed, we can obtain it directly from the construction of $\widetilde{\sigma_{p}^{0}}$ (for instance, see (3.7.2)) with a notice of the fact that $S_{p}=\widetilde{\sigma_{p}^{0}}\left(S_{0}\right)$.)

Returning to the proof of Theorem 2.3, we can apparently assume that $0 \in M$ and $F$ satisfies the normalization in (3.2). Hence, from Proposition 3.6 and the just mentioned argument, the proof of Theorem 2.3 follows.

5. Proof of Theorem 1.1. In this section, we present the proof of Theorem 1.2 and the proof of Theorem 1.1. 
Let $F$ be a proper holomorphic map from $\mathbf{B}^{n}$ into $\mathbf{B}^{N}$, that is $C^{2}$-smooth up to the boundary. Assume that $N<n+P(n, \kappa)$. For each $p \in \partial \mathbf{B}^{n}$. We can find $\Phi_{p}$ and $\Psi_{p}$, biholomorphic linear fractional map from $\mathbf{B}^{n}$ to $\mathbf{S}^{n}$ and $\mathbf{B}^{N}$ to $\mathbf{S}^{N}$, respectively, such that $\Phi_{p}(p)=0$ and $\Psi_{p}(F(p))=0$. Then we define the geometric rank $R k_{F}(p)$ of $F$ at $p$ to be the geometric rank of the map $\Psi_{p} \circ F \circ \Phi_{p}^{-1}$ at 0 . By Lemma $2.2(\mathrm{~B}), R k_{F}(p)$ is a well-defined lower semi-continuous function over $\partial \mathbf{B}^{n}$. Let $\kappa_{0}=\max _{p \in \partial \mathbf{B}^{n}} R k_{F}(p)$. Then $E:=\left\{p: p \in \partial \mathbf{B}^{n}, R k_{F}(p)=\kappa_{0}\right\}$ is an open subset of $\partial \mathbf{B}^{n}$ and $\kappa_{0}<\kappa$ by Lemma 2.2 (B). Now, when $F$ is further assumed to be $C^{3}$, for $p_{0} \in E$, applying Theorem 2.3 to $\Psi_{p} \circ F \circ \Phi_{p}^{-1}$, we conclude that for each $p \in \partial \mathbf{B}^{n}$ close to $p_{0}$, there is a unique affine complex space $S_{p}$, which transversally passes through $\partial \mathbf{B}^{n}$ at $p$ and is of complex dimension $n-\kappa_{0}$ such that the restriction of $F$ to $S_{p} \cap \mathbf{B}^{n}$ is linear. By Theorem 2.3, the collection of all these $S_{p}$ must fill in an open subset $U_{p_{0}}$ close to $p_{0}$ in $\mathbf{B}^{n}$. Apparently, shrinking the size of $U_{p_{0}}$ if necessary, we see that for each $Z=\left(z_{1}, \cdots, z_{n}\right) \in U_{p_{0}}$, there is an affine complex space $S_{Z}$ of dimension $n-\kappa_{0}$ passing through $Z$ such that the restriction of $F$ to $S_{Z}$ is a linear fractional map. Since all affine complex subspaces through $Z$ much intersect $\beta \mathbf{B}^{n}$ near $p$, it follows from Lemma 2.2 (D) that $F$ can not be linear when restricted to any affine subspace through $Z$ of dimension larger than $n-\kappa_{0}$.

Now, to complete the proof of Theorem 1.1, we need only to prove the following:

LEMmA 5.1. Let $F$ be a proper holomorphic map from $\mathbf{B}^{n}$ into $\mathbf{B}^{N}$ with $n \geq 2$. Assume that there is a point $Z_{0} \in \mathbf{B}^{n}$ such that for each $Z \approx Z_{0}$, there is an affine complex subspace $S_{Z}$ of complex dimension $n-\kappa_{0}>0$ such that $\left.F\right|_{S_{Z}}$ is linear fractional. Then $F$ is $\left(n-\kappa_{0}\right)$ - linear over $\mathbf{B}^{n}$.

Proof of Lemma 5.1. Define

$$
\mathcal{P}:=\left\{(Z, S) \in \mathbf{B}^{n} \times G_{n, n-\kappa_{0}}(\mathbf{C}), F \text { is linear fractional when restricted to } Z+S\right\}
$$

We claim that $\mathcal{P}$ is a complex analytic variety. For this purpose, we need to verify that (i) $\mathcal{P}$ is closed; (ii) $\mathcal{P}$ is locally defined by holomorphic functions. To prove $\mathcal{P}$ is closed, let $\left\{\left(Z_{j}, S_{j}\right)\right\} \in \mathcal{P}$ be such that $Z_{j} \rightarrow Z \in \mathbf{B}^{n}$ and $S_{j} \rightarrow S \in G_{n, n-\kappa_{0}}(\mathbf{C})$. After applying an automorphism of $\mathbf{B}^{n}$ if necessary, we can assume, without loss of generality, that $Z=0$ and $S=\operatorname{Span}\left\{e_{1}, \cdots, e_{n-\kappa_{0}}\right\}$. Here $e_{j}$ is the vector in $\mathbf{C}^{n}$, whose component at the $l^{t h}$-position is $\delta_{j}^{l}$. Let $S_{j}=\operatorname{Span}\left\{e_{1}^{j}, \cdots, e_{n-\kappa_{0}}^{j}\right\}$ be such that $e_{l}^{j} \rightarrow e_{l}$ as $j \rightarrow \infty$. Since $F$ is linear fractional when restricted to $S_{j}$, we can write $F\left(Z_{j}+\sum_{j=1}^{n-\kappa_{0}} t_{j} e_{l}^{j}\right)=\frac{F\left(Z_{j}\right)+\sum a_{j}\left(Z_{j}, S_{j}\right) t_{j}}{1+\sum_{j} b_{j}\left(Z_{j}, S_{j}\right) t_{j}}$. Apparently, $a_{j}, b_{j}$ depend continuously on $Z_{j}$ and $S_{j}$. Hence, letting $j \rightarrow \infty$, we see that $F\left(\sum_{j} t_{j} e_{j}\right)$ is linear fractional on $t=\left(t_{1}, \cdots, t_{n-\kappa_{0}}\right)$. We remark that by a result of Alexander, $F$ must be also a biholomorphic map from $S$ into its image which is also an affine space of the same dimension.

Next, we let $\left(Z_{0}, S_{0}\right) \in \mathcal{P}$ and we want to show that $\mathcal{P}$ near $\left(Z_{0}, S_{0}\right)$ is defined by holomorphic equations. As above, we can also assume that $Z_{0}=0$ and $S_{0}=$ $\operatorname{span}\left\{e_{1}, \cdots, e_{n-\kappa_{0}}\right\}$. We will use the standard local coordinates for the Grassmannian $G_{n, n-\kappa_{0}}(\mathbf{C})$ near $S_{0}$. Namely, for any $S$ near $S_{0}$ we associate it uniquely with the coordinates $\left(\xi_{j l}\right)$ where $j$ runs from 1 to $n-\kappa_{0}$ and $l$ runs from $n+1-\kappa_{0}$ to $n$ such that $S=\operatorname{span}\left\{e_{1}(S), \cdots, e_{n-\kappa_{0}}(S)\right\}$. Here $e_{j}(S)=\left(0, \cdots, 1, \cdots, 0, \xi_{j\left(n+1-\kappa_{0}\right)} \cdots, \xi_{j n}\right)$. 
Notice that $(Z, S)\left(\approx\left(0, S_{0}\right)\right) \in \mathcal{P}$ if and only if

$$
F\left(Z+\sum_{j} t_{j} e_{j}(S)\right)=\frac{F(Z)+\sum A_{j}(Z, S) t_{j}}{1+\sum_{j} b_{j}(Z, S) t_{j}}
$$

for certain $N$-tuples $A_{j}^{\prime} s$ and scalar $b_{j}^{\prime} s$, which depend on $(Z, S)$. Write

$$
F\left(Z+\sum_{j} t_{j} e_{j}(S)\right)=\sum_{\alpha} C_{\alpha}\left(Z, \xi_{j l}\right) t^{\alpha}
$$

Then $C_{\alpha}$ depends holomorphically on $\left(Z, \xi_{j l}\right)$. Multiplying $\left(1+\sum_{j} b_{j}(Z, S) t_{j}\right)$ of both sides of (5.1) and then considering the Taylor expansion in $t$ at the origin, we see that (5.1) holds if and only if:

$$
C_{\alpha}+\sum_{j=1}^{n-\kappa_{0}} b_{j} C_{\alpha-e_{j}^{\prime}} \equiv 0 \text { for }|\alpha| \geq 1
$$

$C_{0}=F(Z), C_{e_{j}^{\prime}}=\left.D_{t_{j}} F\left(z+\sum_{j} t_{j} e_{j}(S)\right)\right|_{t=0}$ and $A_{j}=F(Z) b_{j}+C_{e_{j}^{\prime}}$. Here $e_{j}^{\prime}$ is the vector in $\mathbf{C}^{n-\kappa_{0}}$ defined as for $e_{j}$. As we mentioned before, since $\left.F\right|_{S}$ must be a linear embedding, we see that $\left\{C_{e_{j}^{\prime}}\right\}_{j=1}^{n-\kappa_{0}}$ are linearly independent vectors. Hence, we can holomorphically solve $b_{j}^{\prime} s$ in (5.2) in terms of $C_{\alpha}$ with $|\alpha|=1,2$. Hence (5.2) can be completely written as a system of holomorphic equations in $\left(Z, \xi_{j l}\right)$. Together with the closeness of $\mathcal{P}$, we conclude that $\mathcal{P}$ is a complex analytic variety in $\mathbf{B}^{n} \times G_{n, n-\kappa_{0}}(\mathbf{C})$. Let $\pi$ be the natural projection from $\mathcal{P}$ into $\mathbf{B}^{n}$. Then $\pi$ is clearly proper and thus $\pi(\mathcal{P})$ is a subvariety of $\mathbf{B}^{n}$. Since $\pi(\mathcal{P})$ contains an open subset of the ball, we conclude that $\pi(\mathcal{P})=\mathbf{B}^{n}$. Hence, we showed that for each point $Z$ in the ball, there is a complex subspace $S$ of dimension $\left(n-\kappa_{0}\right)$ such that the restriction of $F$ to $S+Z$ is linear. Since a linear fractional map sends affine lines to affine lines we conclude the proof of Lemma 5.1.

Proofs of Theorem 1.1 and Theorem 1.2. Let $\kappa$ be as in Theorem 1.1 and $\kappa_{0}$ be the geometric rank of the map $F$. By Lemma 3.2, $n-\kappa_{0} \geq n-\kappa+1$. Combining Theorem 2.3 with Lemma 5.1, we thus complete the proof of Theorem 1.1.

Meanwhile, by the classical result of Alexander, for any $Z \in \mathbf{B}^{n}$ and any affine subspace $S_{Z}^{a}$ through $Z$ such that $F$ is linear fractional when restricted to $S_{Z}^{a}, F$ must be biholomorphic from $S_{Z}^{a} \cap \mathbf{B}^{n}$ to its image: $A \cap \mathbf{B}^{N}$, where $A$ is a certain affine subspace of $\operatorname{dimension} \operatorname{dim}\left(S_{Z}^{a}\right)$. Theorem 1.2 follows clearly.

The argument presented above, together with Lemma 5.3, can be clearly used to give the following local result of Theorem 1.1:

Corollary 5.2. Let $M$ be a connected open subset of $\mathbf{H}_{n}$ and let $F$ be a $C^{3}$ $C R$ map from $M$ into $\mathbf{H}_{N}$ with $N \geq n>1$. Assume that $F$ extends holomorphically to a sub-domain $\Omega$ of $\mathbf{S}^{n}$ which has $M$ as part of its smooth boundary. Let $\kappa_{0}=$ $\max _{p \in M} R k_{F}(p)$. Then $\left\{p \in M: R k_{F}(p)=\kappa_{0}\right\}$ is an open dense subset of $M$. When $\kappa_{0}<n-1, F$ is $\left(n-\kappa_{0}\right)$-linear over $\Omega$. Furthermore, assume that $F$ has constant geometric rank $\kappa_{0}$ in a connected open subset $M^{\prime}$ of $M$. Then there is a sufficiently small subdomain $\Omega^{\prime}$ of $\Omega$ with $M^{\prime}$ as part of its smooth boundary satisfying 
the following property: For each $Z \in \Omega^{\prime} \backslash E$ with $E$ a certain proper complex analytic variety in $\Omega$, there is a unique complex subspace $S_{Z}$ of dimension $n-\kappa_{0}$ such that the restriction of $F$ to $S_{Z}+Z$ is linear fractional. Also $S_{Z}$, as elements in $G_{n, n-\kappa_{0}}(\mathbf{C})$, depends holomorphically on $Z \in \Omega \backslash E$. Moreover, $S_{Z}$ extends holomorphically to $\Omega^{\prime}$ and continuously up to $M^{\prime}$

LEMMA 5.3. Let $M$ be a connected open subset of $\mathbf{H}_{n}$. Let $F$ be a $C^{2} \mathrm{CR}$ map from $M$ into $\mathbf{H}_{N}$ with $N \geq n>1$ and with constant geometric rank $\kappa_{0}<n-1$. Assume that $F$ extends holomorphically to a sub-domain $\Omega$ of $\mathbf{S}^{n}$, which has $M$ as part of its smooth boundary. Assume that $F$ is $\left(n-\kappa_{0}\right)$-linear over $\Omega$. Let $p_{0} \in M$. Then for $Z(\in \Omega \backslash E) \approx p_{0}$ with $E$ a certain complex analytic variety of positive codimension, there is a unique complex subspace $S_{Z}$ of dimension $\left(n-\kappa_{0}\right)$ such that $F$, when restricted to $S_{Z}+Z$, is linear fractional. Moreover $S_{Z}$, as elements in $G_{n, n-\kappa_{0}}(\mathbf{C})$, depends holomorphically on $Z\left(\approx p_{0}\right) \in \Omega \backslash E$ and extends holomorphically across $E$.

Proof of Lemma 5.3. Without loss of generality, we assume that $p_{0}=0$. For each $p \in M$, write $S_{p}^{a}$ for the unique affine subspace through $p$ of dimension $n-\kappa_{0}$, along which $F$ is approximately linear as in Theorem 3.5. (To be consistent with the notation $S_{Z}$ we use here, we add the superscript $a$ to emphasize $S_{p}^{a}$ is affine.) We can also assume that $S_{0}^{a}$ is the subspace defined by $z_{1}=\cdots=z_{\kappa_{0}}=0$. Since for any $Z(\in \Omega) \approx 0, S_{Z}+Z$ must cut $M$ near the origin, by Theorem 3.5 (B) and Proposition 3.6 (III), $S_{Z}$ as an element in $G_{n, n-\kappa_{0}}(\mathbf{C})$ must be also very close to $S_{0}^{a}$. Let $\mathcal{P}_{r}$ be as defined in (5.0) with $\mathbf{B}^{n}$ being replaced by $\Omega \cap\{|z|<r\}$ for $r<<1$. Still write $\pi$ for the projection from $\mathcal{P}_{r}$ to $\Omega_{r}=\Omega \cap\{|z|<r\}$. Then by the assumption, $\pi$ is a surjective proper map. Since when $r<<1$, for each $Z \in \Omega \cap\{|z|<r\}, \pi^{-1}(Z)$, being close to $S_{0}$, can be embedded into $\mathbf{C}^{\kappa_{0}\left(n-\kappa_{0}\right)}, \pi^{-1}(Z)$ must be a finite set. Present $S_{Z}\left(\approx S_{0}^{a}\right)$ by its coordinates $\left(\xi_{j l}\right)_{1 \leq j \leq n-\kappa_{0}, n+1-\kappa_{0} \leq l \leq n}$ as in the last part of the proof of Lemma 5.1. Let $\mathcal{V}$ be an irreducible component of dimension $n$ of $\mathcal{P}_{r}$. For each $Z \in \Omega_{r}$, write $\pi^{-1}(Z) \cap \mathcal{V}=\left\{S^{(j)}(Z)\right\}_{j=1, \cdots, m}$ with $m$ fixed for a generic choice of $Z$. Let $\sigma_{m, k}$ be the standard symmetric function in $m$ variables of degree $k$. Then for each fixed $(j, l), \sigma_{m, k}\left(\xi_{j l}\left(S^{(1)}(Z)\right), \cdots, \xi_{j l}\left(S^{(m)}(Z)\right)\right)$ is a holomorphic function with boundary value at $p \in M: \sigma_{m, k}\left(\xi_{j l}\left(S_{p}^{a}-p\right), \cdots, \xi_{j l}\left(S_{p}^{a}-p\right)\right)$. Hence, it follows easily that there is only one irreducible component of maximum dimension in $\mathcal{P}$, which must also be single-sheeted and thus is biholomorpic to $\Omega_{r}$ through the projection map $\pi$ away from a proper subvariety. Notice that the other irreducible components of $\mathcal{P}_{r}$ must have projection in $\Omega_{r}$ of positive codimension. By Proposition 3.6 (III) and the Riemann removable singularity theorem, we see the proof of Lemma 5.3.

It would be an interesting open problem to understand how much initial regularity is needed for Theorem 1.1 to hold. This problem, involving the regularity problem of CR mappings with positive codimenions and with minimum initial regularity to start, is known to be much more subtle than the related problems in the equi-dimensional case. (See [Hu2-3] for the references). In passing, the author would like to use this opportunity to give a remark concerning an early paper [Hu3] of the author for the regularity of $\mathrm{CR}$ maps in $\mathbf{C}^{2}$.

REMARK 5.4. There was a misleading sentence on [pp 111, Michigan J. of Math. (51), 2003] by Diederich-Pinchuk claiming that the proof of the main result in [Hu3] was based on ideas of a preliminary version of [Diederich-Pinchuk, Indiana Uinv. 
Math. J. 44 (1995), 1089-1126]. The fact, however, is the opposite- Indeed, a fundamental part of the paper [Diederich-Pinchuk, Indiana Uinv. Math. J. 44 (1995), 1089-1126], (namely, $§ 3-\S 7$, pp 1094-1110), was fundamentally based on the new methods and tools that we had first developed and made public in the earlier circulated preprint $[\mathrm{Hu} 4]$ for solving the regularity problem for proper and CR correspondences. (The interested reader is referred to [pp392-393, Hu2] for more discussions on the historical facts of [Hu3].)

\section{REFERENCES}

[Alx] H. AleXAnder, Proper holomorphic maps in $\mathbf{C}^{n}$, Indiana Univ. Math. Journal, 26 (1977), pp. 137-146.

[BER] M. S. Baouendi, P. Ebenfelt and L. Rothschild, Real Submanifolds in Complex Spaces and Their Mappings, Princeton Univ. Mathematics Series 47, Princeton University, New Jersey, 1999.

[BHR] S. BAOUENDI, X. HUANG AND L. RothSCHILD, Regularity of CR mappings between algebraic hypersurfaces, Invent. Math., 125 (1996), pp. 13-36.

[BT] S. BaOUendi AND F. TReves, A property of the functions and distributions annihilated by a locally integrable system of complex vector fields, Ann. of Math., 113:2 (1981), pp. $387-421$.

[BK] D. BURns AND S. KRANTZ, Rigidity of holomorphic mappings and a new Schwarz lemma at the boundary, Jour. of AMS, 7 (1994), pp. 661-676.

[CaMo] H. CAO AND N. MOK, Holomorphic immersions between compact hyperbolic space forms, Invent. Math., 100 (1990), pp. 49-61.

[CM] S. S. CheRn AND J. K. Moser, Real hypersurfaces in complex manifolds, Acta Math., 133 (1974), pp. 219-271.

[CS1] J. Cima And T. J. Suffridge, A reflection principle with applications to proper holomorphic mappings, Math Ann., 265 (1983), pp. 489-500.

[CS2] J.CIma AND T. J. SUFFridge, Boundary behavior of rational proper maps, Duke Math. J., 60 (1990), pp. 135-138.

[Da1] J. D'Angelo, Several Complex Variables and the Geometry of Real Hypersurfaces, CRC Press, Boca Raton, 1993.

[Da2] J. D'ANGelo, Proper holomorphic mappings between balls of different dimensions, Mich. Math. J., 35 (1988), pp. 83-90.

[Da3] J. D'Angelo, Inequalities from Complex Analysis, Carus Mathematical Monograph, No. 28, Mathematics Association of America, 2002.

[DC] J. D'ANGELo AND D. CATLIn, A stabilization theorem for Hermitian forms and applications to holomorphic mappings, Math Research Letters, 3 (1996), pp. 149-166.

[DL] J. D'Angelo And D. LichtBlaU, Spherical space forms, CR mappings, and proper maps between balls, J. Geom. Anal., 2:5 (1992), pp. 391-415.

[Fa1] J. FARAN, Maps from the two ball to the three ball, Invent. Math., 68 (1982), pp. 441-475.

[Fa2] J. FARAN, On the linearity of proper maps between balls in the lower dimensional case, Jour. Diff. Geom., 24 (1986), pp. 15-17.

[EHZ1] P. EBENFELt, X. HuANG AND D. ZAITSEv, Rigidity of CR submanifolds of spheres, preprint, 2002.

[EHZ2] P. EBENFELt, X. HuANG AND D. ZAITSEv, The equivalence problem and rigidity for hypersurfaces embedded into hyperquadrics, Preprint, 2002. (Amer. Jour. of Math., to appear.)

[Fo1] F. FORSTNERIC, Extending proper holomorphic mappings of positive codimension, Invent. Math., 95 (1989), pp. 31-62.

[Fo2] F. FORSTNERIC, A survey on proper holomorphic mappings, Proceeding of Year in SCVs at Mittag-Leffler Institute, Math. Notes 38 (1992), Princeton University Press, Princeton, N.J.

[HS] M. HaKim And N. Sibony, Fonctions holomorphes bornes sur la boule unite de $\mathbf{C}^{n}$, Invent. Math., 67:2 (1982), pp. 213-222.

[Hu1] X. HUANG, On a linearity problem of proper holomorphic mappings between balls in complex spaces of different dimensions, Jour. of Diff. Geom., 51:1 (1999), pp. 13-33. 
[Hu2] X. HuANG, On some problems in several complex variables and Cauchy-Riemann Geometry, Proceedings of ICCM (edited by S. T. Yau and L. Yang; December, 1998), AMS/IP studies in Advanced Mathematics, 20 (2001), pp. 383-396.

[Hu3] X. HuAng, Schwarz reflection principle in complex spaces of dimension two, Comm. in PDE, 21 (1996), pp. 1781-1828.

[Hu4] X. HUANG, Regularity of holomorphic correspondences and applications to the mapping problem, Preprint publically circulated in August-October of 1995. (This is the early version of [Hu3], whose main content was later published as Part II of [Hu3]).

[HJ] X. HuAng And S. Ji, Mapping $\mathbf{B}^{n}$ into $\mathbf{B}^{2 n-1}$, Invent. Math., 145 (2001), pp. 219-250.

[Low] E. Low, Embeddings and proper holomorphic mappings of strictly pseudoconvex domains into polydiscs and balls, Math Z., 190 (1985), pp. 401-410.

[Mok] NGaiming MoK, Metric rigidity theorems on Hermitian locally symmetric manifolds, Series in Pure Mathematics, 6. World Scientific Publishing Co., Inc., Teaneck, NJ, 1989.

[MSY] N. Mok, Y. T. SIU AND S. K. YEunG, Geometric superrigidity, Invent. Math., 113 (1993), pp. 57-83.

[Mos] J. Moser, Analytic surfaces in $\mathbf{C}^{2}$ and their local hull of holomorphy, Ann. Acad. Sci. Fenn. Ser. A I Math., 10 (1985), pp. 397-410.

[Po] H. PoInCARÉ, Les fonctions analytiques de deux variables et la représentation conforme, Ren. Cire. Mat. Palermo, II. Ser., 23 (1907), pp. 185-220.

[Sp] M. SPIVAK, A comprehensive introduction to differential geometry, Vol. IV. Second edition. Publish or Perish, Inc., Wilmington, Del., 1979.

[Ste] B. Stensones, Proper holomorphic mappings between the balls, Jour. of Geom. Analysis, 6 (1996), pp. 317-339.

[Ta] N. TANAKA, On the pseudo-conformal geometry of hypersurfaces of the space of n complex variables, J. Math. Soc. Japan, 14 (1962), pp. 397-429.

[Tu] ZHEN-HAN TU, Rigidity of proper holomorphic mappings between nonequidimensional bounded symmetric domains, Math. Z., 240:1 (2002), pp. 13-35.

[We1] S. WeBSTER, On mapping an $n$-ball into an $(n+1)$-ball in the complex space, Pac. J. Math., 81 (1979), pp. 267-272.

[We2] S. WebsteR, The rigidity of $C$ - $R$ hypersurfaces in a sphere, Indiana Univ. Math. J., 28:3 (1979), pp. 405-416.

[Yue] C. YUE, Dimension and rigidity of quasi-Fuchsian representations, Ann. of Math., 143:2 (1996), pp. 331-355. 